\title{
Zur Ókologie der Polychaeten des Helgoländer Felslitorals
}

\author{
L. GillandT \\ Zoologisches Institut und Zoologisches Museum; \\ Martin-Luther-King-Platz 3, D-2000 Hamburg 13, Bundesrepublik Deutschland
}

\begin{abstract}
On the ecology of polychaetes of the Helgoland rocky intertidal. The ecology and distribution of the polychaetes inhabiting the rocky intertidal zone of the island of Helgoland (German Bight, North Sea) were studied at five ecologically different stations. Scolelepis squamata, Microphthalmus sczelkowii and Ophryotrocha gracilis were the only typical intertidal polychaetes observed. Of the 53 polychaete species documented, eight were recorded for the first time in the German Bight. Two species and one subspecies had not been described previously. The following inviromental variables were measured and, where possible, related to the occurrence of the polychaetes: $\mathrm{pH}$, redox potential, $\mathrm{O}_{2}$ diffusion rate, temperature, salinity, particle-size distribution, and amount of organic particles. In the investigation area the number of species and their frequency maxima increase horizontally from east to west. This appears to be due to the increasing diversity of microhabitats in the substratum, as well as to conditions of $\mathrm{O}_{2}$ supply or $\mathrm{H}_{2} \mathrm{~S}$ presence. Vertically, the number of species increases with water depth. The potential effects of temperature and salinity on the distribution patterns are discussed.
\end{abstract}

\section{EINLEITUNG}

Die überwiegend meeresbewohnenden Polychaeten sind weltweit verbreitet. Sie haben nahezu alle marinen Biotope besiedelt. Ihre vertikale Verteilung reicht vom obersten Supralitoral bis hinab in Bereiche der Tiefsee. Benthische Polychaeten werden in bzw. auf fast allen Substraten angetroffen: auf Schlick, feinem und grobem Sand und Kies ebenso wie auf allen harten Unterlagen wie Felsen, sekundären Hartböden (Austernbänke, krustenbildende Algen usw.) sowie künstlichen Beton- und Holzbauten. Ebenso wird das Phytal bewohnt.

Polychaeten stellen in weiten Küstenbereichen einen Hauptanteil an Biomasse und bilden ein wichtiges Glied innerhalb der Nahrungsnetze mariner Lebensgemeinschaften. Dennoch sind unsere Kenntnisse über ihr Vorkommen, ihre Verbreitung, ihre ökologischen Ansprüche und ihre Biologie selbst an den Küsten der Nord- und Ostsee noch lückenhaft.

Die vorliegende Untersuchung befaßt sich mit den Polychaeten des felsigen Helgoländer Eulitorals. Dabei wurden auch solche Arten mit erfaßt, die nicht auf oder im Fels selbst leben, sondern das Substrat besiedeln, welches große Teile des Felsuntergrundes bedeckt. Ziele dieser Arbeit waren: (a) eine möglichst vollständige Erfassung der Polychaetenfauna 
des eulitoralen Helgoländer Felswatts, (b) die Untersuchung der Biologie und Okologie der gefundenen Arten, (c) die Analyse der Beziehungen zwischen einigen ausgewählten abiotischen Faktoren und der Verteilung und Abundanz einzelner Polychaetenarten und (d) die taxonomische Bearbeitung der gefundenen Polychaeten.

Die Ergebnisse der taxonomischen Untersuchungen werden an anderer Stelle ausführlich dargestellt (Gillandt, 1979).

Den ersten zusammenfassenden Bericht über die Helgoländer Fauna veröffentlichte Dalla-Torre (1889). Darin führt er u. a. etwa 45 Namen von Polychaetenarten auf, die jedoch z. T. als einander synonym anzusehen sind. Zudem werden nur spärlich Angaben über genaue Fundorte gemacht, so daß diese Aufzählung für einen Vergleich mit den jetzt vorliegenden Sammelergebnissen ungeeignet ist.

Unter dem Themenkreis "Beiträge zur Meeresfauna von Helgoland" erschienen in der Folgezeit eine Reihe von Veröffentlichungen, in denen jeweils eine Tiergruppe bzw. ein Tierstamm behandelt wurden. So bearbeitete Heincke (1896a, b, c) die Fische und Mollusken Helgolands, Hartlaub (1896a, b) die Coelenteraten, Timm (1896) die Copepoden und Cladoceren, Lauterborn (1896) die Protozoen und Rotatorien, Ehrenbaum (1896) die Cumaceen und Schizopoden, Attems (1896) die rhabdocoelen Turbellarien (die acoelen Turbellarien wurden später von Dörjes, 1968, behandelt), Weltner (1896) die Cirripedien, Sokolowsky (1900) die Amphipoden und Hartmeyer (1908) die Ascidien. Die Polychaetenfauna Helgolands ist im Rahmen dieser Reihe nicht bearbeitet worden. Michaelsen (1897) berücksichtigte sie jedoch soweit als möglich in einer Untersuchung über die Polychaeten der deutschen Meere. $\mathrm{Da}$ er jedoch lediglich Dredgefänge - zumeist aus größeren Tiefen - auswerten konnte, die er allerdings durch Museumsmaterial ergänzte, ist ein Vergleich mit der charakteristischen Felslitoralfauna nur bedingt möglich. Das gleiche gilt für die zusammenfassenden Arbeiten von Caspers $(1939,1950)$ über die Helgoländer Fauna.

Untersuchungen, die sich speziell mit der Polychaetenfauna deutscher Küsten befassen, existieren nur in geringer Zahl. So fand Westheide (1966) bei Forschungen im Eulitoral der Insel Sylt 45 Polychaetenarten. Hartmann-Schröder \& Stripp (1968) stellten bei der Auswertung von Benthosproben aus dem südöstlichen Teil der Deutschen Bucht 16 bisher dort nur selten oder noch gar nicht gefundene Polychaetenarten fest.

Weitere Informationen über die Polychaeten unseres Klimabereiches finden sich verstreut in Arbeiten, in denen Wattengebiete unter allgemeinen Gesichtspunkten untersucht wurden. Hier sind insbesondere die Veröffentlichungen von Wohlenberg (1937), Linke (1939) und Schuster (1951) zu nennen; ebenso die Arbeiten von Stripp (1969a, b, c), der mit der Untersuchung der Benthosfauna der Helgoländer Bucht in 10-60 m Tiefe die Kenntnis über die dortige Faunenzusammensetzung beträchtlich erweiterte, und Michaelis $(1970,1975)$, der Studien über die Fauna der Schlick- und Sandwatten bei Schillighörn und in der Wesermündung anstellte.

Über Untersuchungen der Fauna vor ausländischen Küsten existieren zahlreiche Publikationen. Doch wurden diese Arbeiten entweder im sublitoralen Bereich ausgeführt: Eliason (1920), Bruce, Colman \& Jones (1963), Hamond (1966), Bellan (1969), Kirkegaard (1969) und George (1974), oder behandeln Strände mit Feinsubstrat: Mortensen (1922), Amoureux (1966), Amanieu (1969) und Gibbs (1969), oder aber sie stammen aus völlig anderen Klimabereichen: Amanieu (1969), Amoureux \& Katzmann (1971), Gruet (1974). 
Damit bietet keine dieser Arbeiten die Möglichkeit eines direkten Vergleichs mit der zur borealen Klimazone gehörenden eulitoralen Polychaetenfauna des Helgoländer Felswatts.

\section{DAS UNTERSUCHUNGSGEBIET}

\section{Lage, Entstehung und Gliederung}

Die Hauptinsel von Helgoland besteht aus unterschiedlich harten und verschieden wiederstandsfähigen Schichten des. Mittleren Buntsandsteins. Charakteristisch für Helgoland ist die Schräglage dieser Buntsandsteinschichten, die eine Folge der Aufwölbung und anschließender Kippung um $10^{\circ}-20^{\circ}$ aus der Horizontalen eines unter Helgoland liegenden Salzstockes während des Tertiärs ist (Hagmeier, 1930; Schmidt-Thomé, 1937).

Durch Verwitterungseinflüsse unterschiedlicher Art, auf die hier nicht näher eingegangen werden soll (vgl. hierzu Krumbein, 1975, 1977), sind die Felsschichten allmählich so weit abgetragen worden, daß heute nur noch ein verhältnismäßig kleiner Teil als steil abfallender Landsockel über dem Meeresspiegel liegt. Am Fuße des Sockels folgt als nächsttiefere Stufe die Abrasionsterrasse, die im Süden, Westen und Nordosten die Insel umgibt. Der oberste Teil dieser Terrasse, die als Felsbankfläche ausgebildet ist (Hagmeier, 1930), fällt in Abhängigkeit von den Gezeiten regelmäßig trocken und wird als "Felswatt" im engeren Sinn bezeichnet.

Das am Fuß der Steilküste gelegene Hauptuntersuchungsgebiet (Abb. 1) wird durch die Abrasionsterrasse gebildet, die im Verlauf der sie landwärts begrenzenden, etwa $500 \mathrm{~m}$ langen Küstenlinie unterschiedlich breit ist. Ihre größte Ausdehnung erreicht sie nach Nienburg (1930) im Norden der Insel, wo die "Nodhurnklippen" bei Niedrigwasser mehrere hundert Meter Entfernung von der Steilküste trockenfallen können. Das Gebiet wird im Nordwesten durch die Nordmole begrenzt.

Das westlich davon gelegene Watt fiel im Untersuchungszeitraum vom Januar 1973 bis Dezember 1974 so selten trocken, daß es nur ausnahmsweise zu begehen war und daher nicht in die Untersuchung einbezogen wurde. Im Osten schließt sich an das Untersuchungsgebiet ein - auf den ersten Blick recht einförmiger - Sand- und Geröllstrand an. Dieser wurde wegen seiner unterschiedlichen $\mathrm{H}_{2} \mathrm{~S}$-Verhältnisse, durch welche die Verteilung der Polychaeten beeinflußt wird, bei der Erfassung einiger Polychaetenarten (Nereis und Malacoceros) berücksichtigt. Die seewärtige Begrenzung des Untersuchungsgebietes ist nicht exakt zu lokalisieren. Sie wechselt mit dem jeweiligen Gezeitenstand. Seine maximale Ausdehnung erreicht das Gebiet bei Nipp-Tiden, bei denen selbst Bereiche mit Laminaria-Vegetation trockenfallen.

In Anlehnung an Remane (1940) und Stephenson \& Stephenson (1972) ist die vertikale Zonierung des Helgoländer Felswatts in leicht schematischer Form wie folgt zu beschreiben:

S u p ralit or a 1 (bzw. Supralitoralsaum): der am weitesten landwärts gelegene Strandabschnitt, der nur bei extremem Hochwasser überspült wird, meist jedoch trocken liegt und üblicherweise nur von Spritzwasser beeinflußt wird. Littorina saxatilis und Littorina littorea besiedeln diese Zone gerade noch. Eine eigene Flora fehlt. Gelegentlich sind angeschwemmte Algen zu finden. 


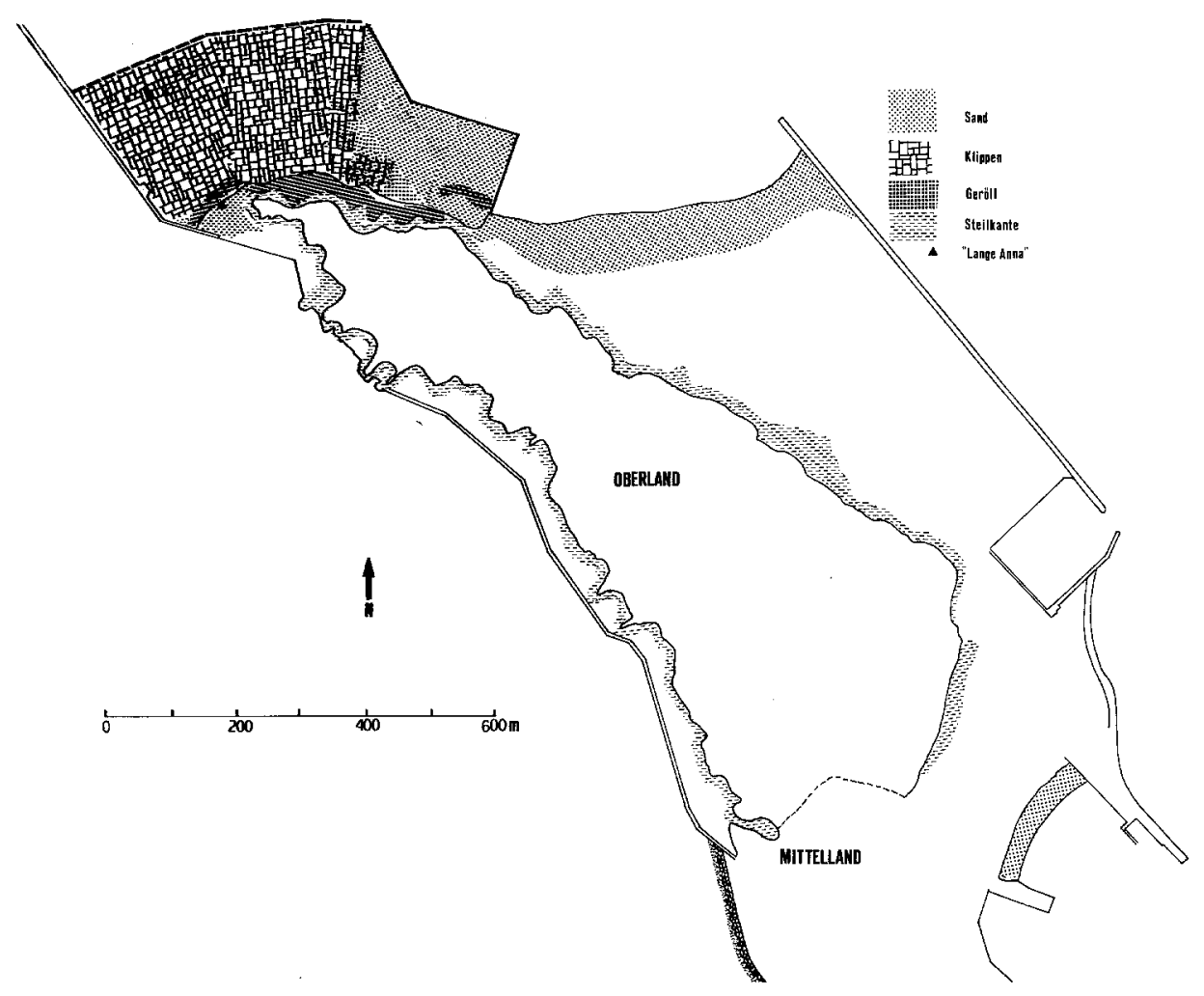

Abb. 1: Lage des Untersuchungsgebietes auf Helgoland

Euli to r a l: der eigentliche Gezeitenbereich, der zweimal täglich trockenfällt. Wegen der charakteristischen Phytalabfolge bietet sich für Helgoland eine weitere Unterteilung des Eulitorals an in: (a) oberes Eulitoral (der am längsten trockenliegende Bereich; typischer Algenbewuchs: Enteromorpha lingulata; ausgezeichnet durch extreme Artenarmut; bewohnbar nur für Tiere, die vorübergehende Aussüßung durch gelegentliche starke Regenfälle tolerieren), (b) mittleres Eulitoral (der dem oberen Eulitoral seewärts folgende Bereich; charakteristischer Bewuchs: Fucus vesiculosus und Fucus serratus; gegenüber der vorherigen Zone erheblich größerer Artenreichtum) und (c) unteres Eulitoral (unterster Abschnitt des Gezeitenbereiches; oberste Grenze von Laminaria saccharina; weiter steigende Artenzahlen).

$\mathrm{S} \mathrm{u}$ b li t o r a l : gewöhnlich stets vom Wasser bedeckter Bereich mit starkem Laminaria-Bewuchs. Gelegentliches Trockenfallen des obersten Abschnittes, der nach Stephenson \& Stephenson (1972) als "Sublitoralsaum" bezeichnet wird, erlaubte sehr selten auch das Sammeln reiner Sublitoralfauna in der Originalzone. Sublitorale Polychaetenarten ließen sich regelmäßig auch in stehenbleibenden Gezeitentümpeln des tiefer gelegenen Eulitorals sowie in den Rhizoiden angespülter Laminarien finden. 


\section{Sammelabschnitte}

Wegen der Beeinflussung des Untersuchungsgebietes durch Ebbe und Flut, durch die sich einzelne Biotope infolge starker Substratumlagerung, Versetzung von Geröll und Steinbrocken von einem Monat zum nächsten völlig verändern konnten, erwies es sich als unzweckmäßig, feste, genau lokalisierte Sammelstationen zu errichten. Statt dessen wurde das gesamte Gebiet von Osten nach Westen in fünf horizontal aufeinanderfolgende, senkrecht zur Küstenlinie verlaufende Sammelabschnitte unterteilt, die sich (bis auf die Sammelabschnitte 4 und 5, die sich zumindest im unteren Teil gleichen) bezüglich ihrer ökologischen Faktoren deutlich voneinander unterschieden (vgl. Skaumal, 1977).

$\mathrm{Sammelabschnitt} \mathrm{1:} \mathrm{Der} \mathrm{obere} \mathrm{Teil} \mathrm{dieses} \mathrm{Abschnittes} \mathrm{am} \mathrm{östlichen} \mathrm{Ende}$ des Gebietes besteht aus einer freiliegenden Sandfläche, die, da sie einer starken Umlagerung unterworfen ist, in wechselnder Schichtdicke den darunter befindlichen Felsboden bedeckt. So wurde nach Stürmen im November 1973 fast das gesamte Substrat abgetragen. Lediglich die Substratoberfläche war in diesem Gebiet gelegentlich oxidiert. Bereits in 1-2 $\mathrm{cm}$ Tiefe war der Sand infolge von $\mathrm{H}_{2} \mathrm{~S}$-Bildung stets tiefschwarz. Nur die Wohnröhren des Polychaeten Malacoceros fuliginosus hoben sich als senkrechte helle Streifen vom Faulschlamm ab. Vermutlich gibt es für die $\mathrm{H}_{2} \mathrm{~S}$-Bildung an dieser Stelle mehrere Ursachen. Zum einen werden hier regelmäßig, besonders bei rauher See nach Nordweststürmen, abgerissene Algen - vor allem Laminarien - angespült und bleiben in mehr oder weniger dicken Schichten liegen. Diese Ablagerungen werden mit Sand bedeckt und beginnen zu faulen. Zum anderen trug sicherlich auch die im Untersuchungsraum am Strand bestehende Müllkippe, von der das Regenwasser nach dem Durchsickern des Abfalls bereits schwarz gefärbt zum Meer fließt, an dieser Stelle zur Schwefelwasserstoffbildung bei. Ob die Annahme Skaumals (1977) zutrifft, wonach die $\mathrm{H}_{2} \mathrm{~S}$-Bildung im Abschnitt 1 auf die Verdriftung häuslicher Abwässer von der Einleitungsstelle nördlich des Kurmittelhauses in das Untersuchungsgebiet zurückzuführen ist, konnte nicht geklärt werden und erscheint fraglich. Chrarakteristische Polychaetenarten im Faulschlamm sind Malacoceros fuliginosus und Capitella capitata. Seewärts wird die Sandfläche durch ein Geröllfeld mit größeren Steinen und den Überresten einer alten Geleiskörperanlage abgelöst. Die sich unterhalb anschließenden Felsklippen fielen im Untersuchungszeitraum selbst bei starkem Niedrigwasser kaum jemals trocken.

Sammelabschnitt 2: Im oberen Teil von Abschnitt 2 ist ebenfalls ein allerdings schmalerer - Sandstreifen auf dem Felsuntergrund zu finden, der jedoch stärker mit Geröllbrocken durchsetzt ist als im Abschnitt 1. Schwefelwasserstoff wird kaum angetroffen. Seewärts ist die gleiche Geröllzone wie im Abschnitt $1 \mathrm{zu}$ finden. Vor der Niedrigwasserlinie existiert ein größerer Gezeitentümpel. Vorhandene Felskippen gehören ebenso wie beim Abschnitt 1 dem Sublitoral an und fallen nur ausnahmsweise trocken.

$\mathrm{S}$ a m m e la b s chn it t 3 : Dieser Abschnitt unterscheidet sich erheblich von den beiden ersten. Der obere Teil wird aus einem bis zum Grund oxydierten Sand- und Schillsubstrat gebildet, in dem die zahlreichen Trümmersteine eines alten Bunkers liegen. In einer Tiefe zwischen ca. 60-100 cm stößt man auf eine Lehm-Ton-Schicht. Die charakteristische Polychaetenart dieses Bereiches ist Scolelepis squamata. Seewärts folgt dem Sandstück eine sich zungenförmig ins Meer vorschiebende Fläche aus grobem Substrat, Geröll und zum Teil durch Bitumen miteinander verbundenen Steinblöcken, die 
wegen ihrer dichten Besiedlung mit Mytilus edulis und einigen Littorina-Arten auch als "Miesmuschelbank" bezeichnet wird. Einige kleinere Rockpools in diesem Gebiet werden von Lanice conchilega bewohnt. In Höhe des dritten Abschnitts wird - von Osten nach Westen gesehen - bei starkem Niedrigwasser erstmalig regelmäßig ein kleines Stück der Klippen sichtbar, die charakteristisch für die beiden letzten Sammelabschnitte im äußersten Westen des Untersuchungsgebietes sind.

$\mathrm{S}$ a m m e la b s h n i t 4: Unterhalb der Steilkante beginnt dieser Abschnitt mit großen, leicht schräg zum Meer geneigten Felsplatten, die nur gelegentlich mit einem dünnen Algenüberzug versehen, sonst aber völlig unbedeckt und auch frei von Substrat sind. Auf ihnen befinden sich einige große Felsblöcke. Der untere Teil besteht aus den schon mehrfach erwähnten Klippen. Bei diesen Klippen handelt es sich um senkrecht zum Strand verlaufende, langgestreckte, stark zerklüftete Felsrücken, die aus ihrer Umgebung hervorragen. Sie sind nach Hagmeier (1930) Reste von härteren aufgerichteten Gesteinsschichten, aus denen weichere Gesteine durch Abtragung und Verwitterungseinflüsse ausgeräumt wurden.

Hagmeier (1930) unterscheidet folgende zu den Klippen gehörende Oberflächengebilde (Abb. 2): (a) Schichtflächen (die ursprünglichen Oberseiten der Sedimentschichten, die den Brandungseinflïssen voll ausgesetzt sind), (b) Schichtköpfe (die vorstehenden Kanten harter, übriggebliebener Gesteinsplatten, die bei der Entstehung Helgolands aus der ursprünglichen Horizontalen in die bereits erwähnte Schräglage gebracht wurden), (c) Schichthöhlen (geschützte Höhlungen, die durch die Erosion weicher Schichten unter härteren, stehenbleibenden entstehen), (d) Mulden oder Längsgräben (die Einsenkung zwischen zwei Schichtköpfen), (e) Querrinnen und Priele (ausgewaschene Verwerfungsspalten) und (f) Felstümpel (Vertiefungen von weichen Muldenstellen, die meistens an Kreuzungspunkten von Längs- und Quergräben entstehen).

In den Mulden, Rinnen und Gezeitentümpeln zwischen den Klippen bleibt gewöhnlich auch während der Ebbezeit Wasser stehen. Damit haben Organismen, die üblicher-

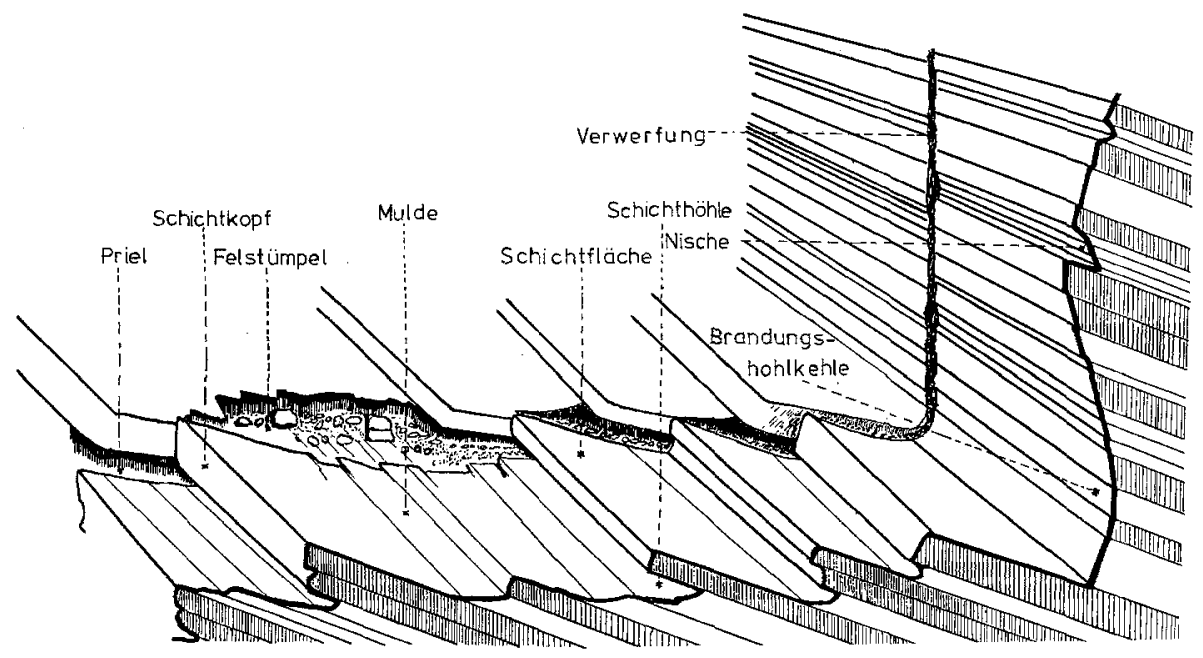

Abb. 2: Schematische Darstellung der Oberflächenstruktur des Helgoländer Felslitorals. (Verändert nach Hagmeier, 1930) 
weise im Sublitoral vorkommen, die Möglichkeit, auch im Eulitoral Bereiche mit ihnen gemäßen Lebensbedingungen aufzusuchen und zu besiedeln.

Durch die starke Zerklüftung und Zergliederung des Felswatts wird die Bildung einer großen Zahl von Kleinbiotopen begünstigt, die Organismen mit unterschiedlichsten ökologischen Ansprüchen einen Siedlungsraum bieten. In diesem Zusammenhang sind vor allem die geschützt liegenden Spalten, Nischen und Schichthöhlen von Bedeutung, die zumeist mit Ablagerungen wie Sand, Schill, Verwitterungsprodukte des Untergrundes sowie Detritus angefüllt sind und auch empfindlichen Tieren die Besiedlung erlauben.

$\mathrm{S}$ a m mela bschnit 5 : Dieser Abschnitt unterscheidet sich von dem vorhergehenden im unteren Teil lediglich durch seine größere seewärtige Ausdehnung. Zum Land hin besteht er aus einer Sand- und Schillfläche, auf der sich als großer einzeln stehender Felsen die "Lange Anna" erhebt. In diesem Substrat, das dem aus Sammelabschnitt 3 ähnelt, fehlt Scolelepis squamata. Die steiler abfallende Neigung zum Meer sowie die verglichen mit Abschnitt 3 - ungeschütztere Lage mögen Gründe hierfür sein.

\section{MATERIAL UND METHODE}

Außer bei einer Voruntersuchung im Oktober 1972 erfolgte die Probeentnahme im Zeitraum vom Januar 1973 bis Dezember 1974 in ungefähr monatlichen Abständen während eines jeweils mehrtägigen Aufenthaltes in der Biologischen Anstalt auf Helgoland. Die Aufenthalte richteten sich dabei nach tageszeitlich günstig gelegenen Niedrigwasserständen. Da der Wasserstand von Mal zu Mal stark schwanken konnte, war es nicht immer möglich, Proben aus den tiefer gelegenen Wattregionen zu entnehmen.

\section{Entnahme und Bearbeitung der Proben}

Proben wurden während jedes Aufenthaltes in allen fünf Sammelabschnitten genommen, wobei vom oberen Teil aus dem zurückweichenden Wasser folgend nach Möglichkeit alle Zonierungen berücksichtigt wurden.

Für verschiedene Substrate erwiesen sich unterschiedliche Sammelmethoden als notwendig, um eine möglichst vollständige Erfassung der Polychaetenfauna zu gewährleisten. Auf eine quantitative Probenahme - etwa mit einem Stechrohr von definierter Oberfläche die theoretisch zumindest in den Sand- und Schillflächen möglich gewesen wäre, wurde wegen der häufig wechselnden Schichtdicke des Substrates verzichtet.

Proben von weichem Substrat wurden mit Hilfe einer kleinen Schaufel in PlastikSchraubdeckelgefäße gefüllt. Substrat wurde sowohl von freiliegenden Flächen als auch von geschützten Stellen an bzw. unter Felsbrocken entnommen. Die Unterseiten von hochgenommenen Steinblöcken wurden mit einer Pinzette abgesammelt bzw. von anhaftendem Substrat durch Abschaben befreit.

Im Klippenbereich wurden Sand- und Schillablagerungen aus geschützten Spalten und Höhlungen herausgekratzt. Durch Hochbrechen von Felsplatten des Buntsandsteines 
konnte auch der Lebensraum zwischen den einzelnen Schichtflächen zugänglich gemacht werden. Die hier im Gestein bohrenden Polychaeten wurden - soweit es sich um große Formen handelte - einzeln gesammelt. Kleinere Formen wurden zusammen mit Stücken von Schichtplatten eingebracht.

Röhrenbauende Polychaeten auf Pflanzenoberflächen wurden mit den entsprechenden Pflanzenteilen gesammelt. Fucus- und vor allem Laminaria-Rhizoide aus Gezeitentümpeln wurden mit den ihnen anhaftenden Gesteinsunterlagen eingebracht.

Waren die tieferen Teile des Felswatts wegen zu hohen Wasserstandes nicht erreichbar, so wurden auch gelegentlich frisch am Strand angespülte Laminaria-Rhizoide aus dem Sublitoral gesammelt. Alle Proben wurden in Plastikgefäßen zur weiteren Bearbeitung ins Labor geschafft.

Weichsubstratproben wurden in eine etwa $20 \times 30 \times 3 \mathrm{~cm}$ große Kunststoffschale gebracht und größere Polychaetenexemplare zunächst makroskopisch ausgesucht. Da die Tiere beim direkten Úberführen in eine Fixierlösung leicht in mehrere Teile autotomierten, wurden sie zuvor in einem Gefäß mit Seewasser mit $\mathrm{MgCl}_{2}$ bzw. MS 222 (Firma Sandoz) betäubt, wobei sich das letztgenannte Mittel wegen seiner schnellen Wirkung als besonders günstig erwies (vgl. Orrhage, 1967). Anschließend wurden die Proben portionsweise mit Seewasser aufgeschlämmt und mehrfach durch ein Gazenetz von $100 \mu$ dekantiert. Diese geringe Maschenweite war notwendig, um auch die im Sand lebenden Kleinformen zu erfassen. Der Siebrückstand wurde danach in Petrischalen unter dem Binokular ausgesucht, wobei kleine Polychaeten unter $10 \mathrm{~mm}$ Länge abpipettiert und direkt in $70 \%$ igen Alkohol überführt werden konnten, ohne zu autotomieren.

Hartsubstratproben, wie durchlöcherte Gesteinsbrocken, Stücke von losgebrochenen Buntsandsteinschichten und Laminaria-Rhizoide wurden in ein Gefäß mit abgestandenem Seewasser, dem einige Tropfen $40 \%$ iges Formol zugesetzt worden waren, überführt. Bedingt durch die folgende Klimaverschlechterung verließen viele Polychaeten innerhalb weniger Stunden ihren Aufenthaltsort und waren leicht abzusieben.

Um auch die im Substrat verbliebenen Exemplare zu erfassen, wurden die Gesteine anschließend vorsichtig mechanisch zerkleinert, die sich in ihnen noch befindenden Würmer langsam, um ein Zerreißen zu vermeiden, herausgezogen und wie oben beschrieben fixiert. Laminaria-Rhizoide wurden zerschnitten und in kleinen Stücken unter dem Binokular auf verbliebene Polychaeten untersucht. Alle Rückstände wurden danach mehrfach ausgewaschen, durch ein Gazenetz dekantiert und nochmals ausgesucht.

Die Kalkbauten von röhrenbauenden Polychaeten wurden zum Teil vorsichtig von ihrer Unterlage (Gestein oder Pflanzenteil) gelöst, zum Teil mit kleinen Unterlagestücken fixiert.

Alle so erhaltenen Polychaeten wurden nach Proben getrennt in Glasgefäßen mit $70 \%$ igem Alkohol bis zur systematischen Weiterbearbeitung aufbewahrt.

Die Bestimmung der Polychaeten (vgl. Gillandt, 1979) erfolgte nach Fauvel (1923, 1927), Friedrich (1938) und vor allem Hartmann-Schröder (1971). Etwaige zur Determination notwendige Präparationen von Elytren, Parapodien, Kiefern etc. wurden unter dem Binokular vorgenommen. Die Präparate wurden in Glycerin gebettet, unter dem Interferenz-Kontrast-Mikroskop untersucht und zum Teil gezeichnet. Kleinere Polychaeten (bis maximal $5 \mathrm{~mm}$ Länge) wurden vollständig in Glycerin eingebettet untersucht. Bestimmte Oberflächenstrukturen einzelner Arten wurden zudem unter einem Cambridge-Stereo- 
Scanning-Raster-Elektronenmikroskop S 4 fotografiert (Leihgabe der Deutschen Forschungsgemeinschaft).

\section{Erfassung abiotischer Faktoren}

Um den Lebensraum der Polychaeten (Substrat, Wasser, Luft) näher zu charakterisieren, wurden folgende Faktoren bestimmt: (a) Substrat: pH-Wert, Redoxpotentialverteilung, Sauerstoffdiffusionsrate; diese Messungen wurden mit tragbaren Geräten (Fa. Knick) mittels Einstichelektroden direkt am Ort der Probeentnahmen durchgeführt. Im Labor wurden zusätzlich die Korngrößenverteilung sowie der Gehalt an partikulärer organischer Substanz (durch Glühverlust) bestimmt. (b) Wasser: pH-Wert, Temperatur und Salinität. (c) Luft: Temperatur.

\section{ERGEBNISSE}

$$
\mathrm{pH}-\mathrm{Wert}
$$

Der $\mathrm{pH}$-Wert übt für sich betrachtet keinen entscheidenden Einfluß auf die Verteilung von Organismen aus (Delamare-Deboutteville, 1960; Renaud-Debyser, 1963; Giere, 1971; Hartwig, 1973; Pfannkuche, 1977). In Verbindung mit hohen Temperaturen jedoch können hohe $\mathrm{pH}$-Werte für die Organismenverteilung als limitierender Faktor von Bedeutung sein (Wieser, 1975).

$\mathrm{pH}-$ Wert-, $\mathrm{O}_{2}$ - und Redox-Messungen konnten aus technischen Gründen - zum einen standen die Geräte, die von mehreren Personen gleichzeitig benutzt wurden, nicht immer zur Verfügung, zum anderen waren die Elektroden einige Male defekt - nicht in jedem Monat vorgenommen werden. Sie wurden zudem nur in den Sammelabschnitten 1 und 3 durchgeführt, da nur hier regelmäßig einheitliches Substrat zu finden war. Die Geröllbrocken, die das Substrat im Abschnitt 2 durchsetzten, behinderten die Messungen mit den empfindlichen Glaseinstichelektroden.

Die Abbildung 3a und b zeigt den Verlauf der $\mathrm{pH}$-Werte im Untersuchungszeitraum im Sammelabschnitt 1 und 3 sowohl ar der Sedimentoberfläche als auch in etwa $5 \mathrm{~cm}$ Tiefe.

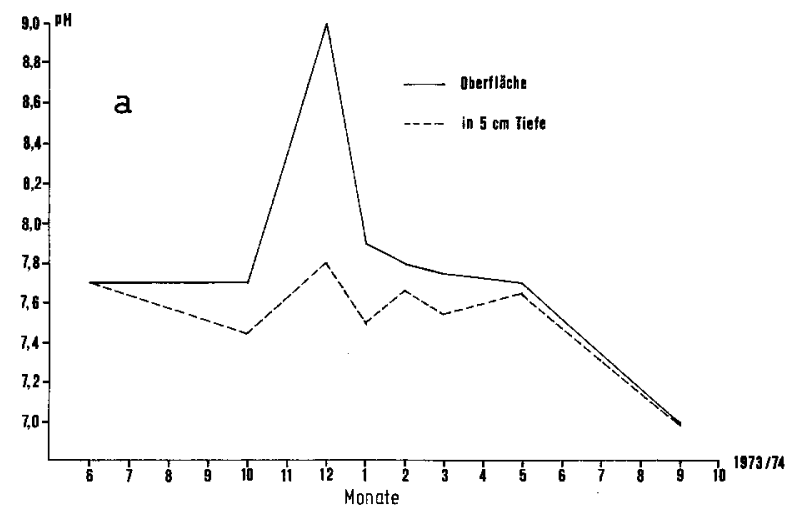

Abb. 3: pH-Wert im Jahresverlauf: Sammelabschnitt 1(a) und Sammelabschnitt 3(b). 


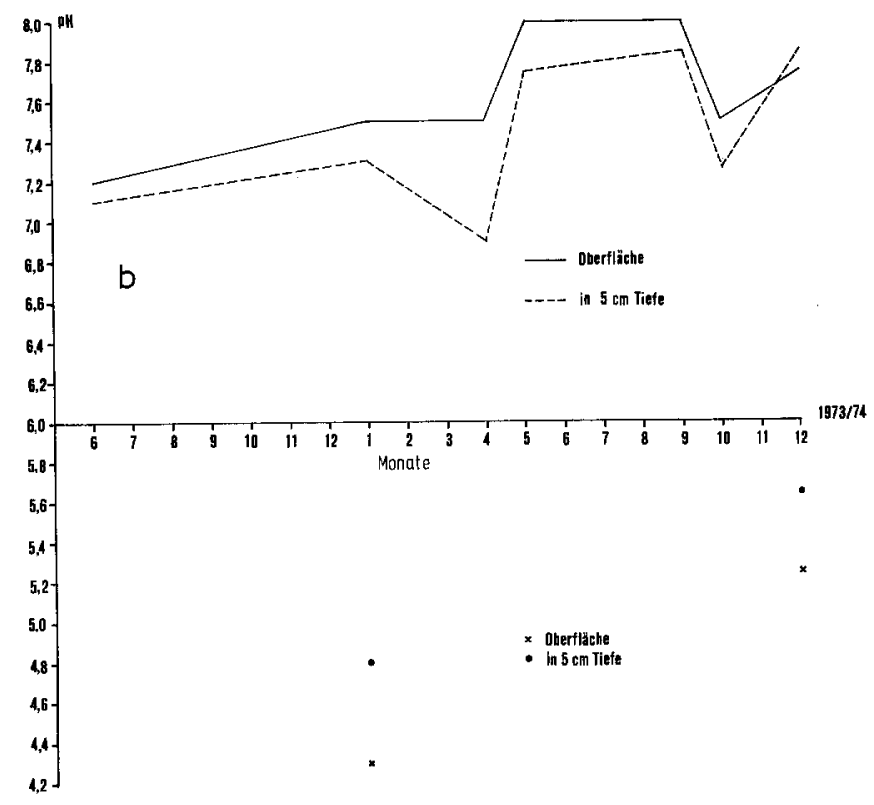

Der im Dezember 1973 im Abschnitt 1 gemessene auffallend hohe Wert von 9 hängt vermutlich mit dem Auftreten einer Diatomeenblüte zusammen. Die Werte in $5 \mathrm{~cm}$ Tiefe liegen erwartungsgemäß - da sie in reduzierendem Medium gemessen wurden - ausnahmslos unter den entsprechenden an der Oberfläche.

Die Werte im Abschnitt 3 liegen insgesamt etwas mehr zum sauren Bereich verschoben und sinken bis auf 6,9 , obwohl hier auch in $5 \mathrm{~cm}$ Tiefe keine reduzierenden Bedingungen herrschen. Die völlig aus dem Rahmen fallenden Werte von 4,2 (4,7) und 5,3 $(5,7)$ wurden unter Trümmerresten eines alten Bunkers gemessen. Da im Gegensatz zu allen anderen Fällen der niedrigere Wert hier an der Oberfläche gefunden wurde, kann vermutet werden, daß die hohe Acidität auf saure Verwitterungsprodukte der Trümmer zurückzuführen ist. Die $\mathrm{pH}$-Werte im freien Wasser wurden mir freundlicherweise von der Biologischen Anstalt Helgoland zur Verfügung gestellt. Sie schwanken zwischen $\mathrm{pH} 8$ und 8,4 und liegen somit deutlich über den Werten im Substrat (vgl. hierzu Schmidt, 1968).

\section{Die Sauerstoffverhältnisse im Sediment}

Die Verteilung vieler Organismen wird in starkem Maße von den Sauerstoffverhältnissen im Sediment beeinflußt (Fenchel \& Jansson, 1966; Fenchel, Jansson \& von Thun, 1967; Jansson, 1967; Enckell, 1968; Giere, 1971, 1973).

Da für die direkte $\mathrm{O}_{2}$-Bestimmung im Sediment bisher keine brauchbare Meßmethode existiert, sind indirekte Methoden anzuwenden, die Rückschlüsse auf die Sauerstoffverhältnisse im Substrat gestatten. Hierfür ist die Messung von Redoxpotentialen geeignet, die eine Vielzahl der komplexen Redoxvorgänge im Sediment erfaßt und sich als hilfreich bei der Beurteilung eines Biotops bezüglich seiner Sauerstoffversorgung erwiesen hat (Zobell, 
1946; Wood, 1965; Fenchel, 1971). Redoxpotentiale (RDP) werden als Eh-Werte in $\mathrm{mV}$ angegeben.

Hohe positive Eh-Werte (über $+150 \mathrm{mV}$ ) zeigen üblicherweise das Vorhandensein von Sauerstoff im Sediment an (Giere, 1973), während negative Eh-Werte mit dem Auftreten von $\mathrm{H}_{2} \mathrm{~S}$ und dem Fehlen von $\mathrm{O}_{2}$ korreliert sind (Fenchel \& Jansson, 1966; Whitfield, 1969). Die Reduktion von Schwefel beginnt bereits ab $+100 \mathrm{mV}$ (Baas-Becking \& Wood, 1955; Fenchel \& Jansson, 1966; Fenchel \& Riedl, 1970). Bei Eh-Werten von unter $-100 \mathrm{mV}$ kann nach Wieser (1975) die Anwesenheit von freiem molekularem Sauerstoff ausgeschlossen werden.

Als weiteres Hilfsmittel zur Abschätzung der Sauerstoffverhältnisse im Sediment wurde die von Lemon \& Erickson $(1952,1954)$ entwickelte Methode der Messung der Sauerstoffdiffusionsrate (ODR) mit einer Platinelektrode benutzt. Dieses Verfahren mißt ebenfalls keine $\mathrm{O}_{2}$-Konzentration, erfaßt jedoch aus dem Komplex der außerdem im Substrat auftretenden Diffusionsvorgänge nahezu ausschließlich die Sauerstoffdiffusion. ODR-Werte werden in $\mathrm{g} \mathrm{O}_{2} \times 10^{-6} \times \mathrm{cm}^{-2} \times \mathrm{min}^{-1}$ angegeben.

Für $\mathrm{O}_{2}$-arme, $\mathrm{H}_{2} \mathrm{~S}$-reiche Sedimente liegen die ODR-Werte bei 0,1 . und darunter (Giere, 1973). Eine unmittelbare Korrelation zwischen Eh- und ODR-Werten existiert nach Enckell (1968) nicht. Dennoch sind hohenegative Eh-Werte oft gekoppelt mit niedrigen ODR-Werten (um null) (Giere, 1973). Keine der beiden Methoden ist frei von Schwächen (Enckell, 1968; Fenchel, 1969; Schmidt \& Machan, 1975); dennoch erscheint es sinnvoll, sie zur Beurteilung der $\mathrm{O}_{2}$-Verhältnisse eines Sedimentes zu kombinieren, zumal es bisher keine besseren Methoden für In-situ-Messungen von $\mathrm{O}_{2}$ gibt.

Die Abbildungen 4 bis 7 zeigen den vertikalen Verlauf der Redox- und ODR-Werte in den Sammelabschnitten 1 und 3. In allen Kurven ist ein starkes vertikales Gefälle zu erkennen. Im Abschnitt 1 weist nur die Substratoberfläche positive Eh-Werte auf (Abb, 4). Bereits in $1 \mathrm{~cm}$ Tiefe läßt der Redox-Wert von $-250 \mathrm{mV}$ auf ein absolut sauerstofffreies Milieu schließen. In den Wohnröhren von Malacoceros wurden ebenfalls nur negative Redoxpotentiale gemessen, wenn diese auch bei weitem nicht so hoch liegen wie im

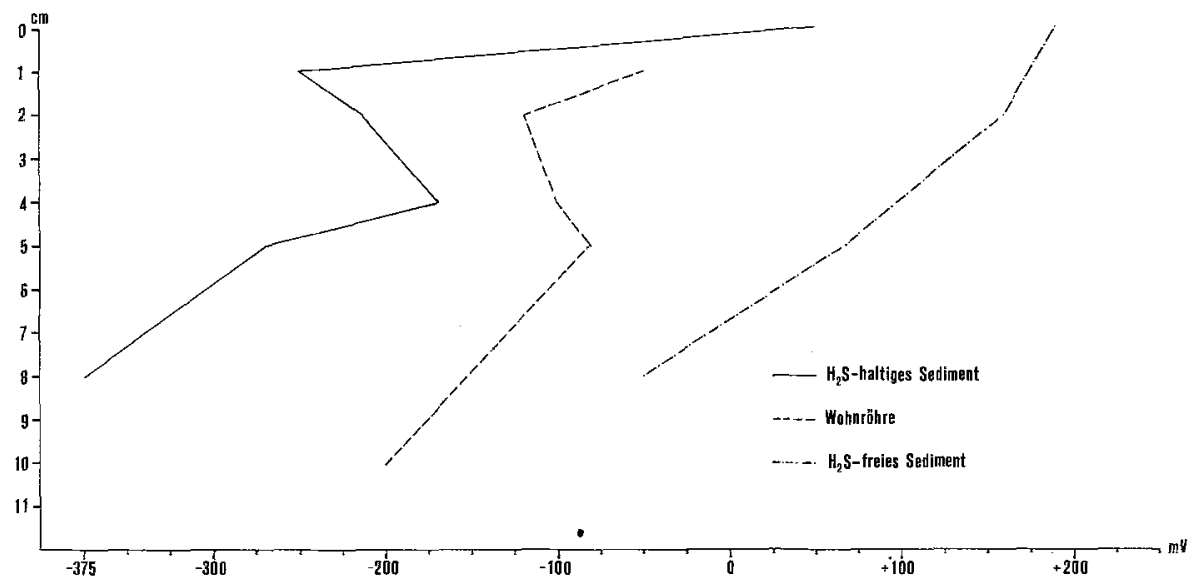

Abb. 4: Vertikaler Verlauf der Redoxpotentiale im Sammelabschnitt 1 im $\mathrm{H}_{2} S$-Sediment, in Wohnröhre und im $\mathrm{H}_{2} \mathrm{~S}$-freien Sediment (Dezember 1973) 
umgebenden $\mathrm{H}_{2} \mathrm{~S}$-haltigen Sediment. Lediglich im Dezember 1973 reichte nach starken Stürmen, die mit einer Sedimentumlagerung einhergingen, die Oxidationszone etwa $5 \mathrm{~cm}$ tief. Der niedrigste Eh-Wert wurde mit $-375 \mathrm{mV}$ in $8 \mathrm{~cm}$ Tiefe gemessen.

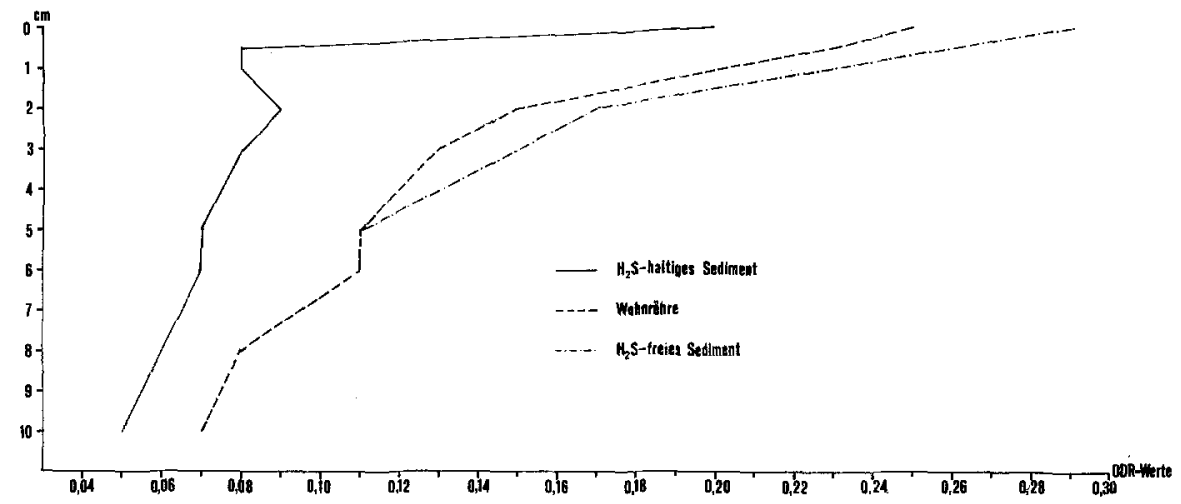

Abb. 5: Vertikaler Verlauf der ODR-Werte $\left(\mathrm{O}_{2}\right.$-Diffusionsrate) im Sammelabschnitt 1 im $\mathrm{H}_{2} \mathrm{~S}$ Sediment, in Wohnröhre und im $\mathrm{H}_{2} \mathrm{~S}$-freien Sediment (Dezember 1973)

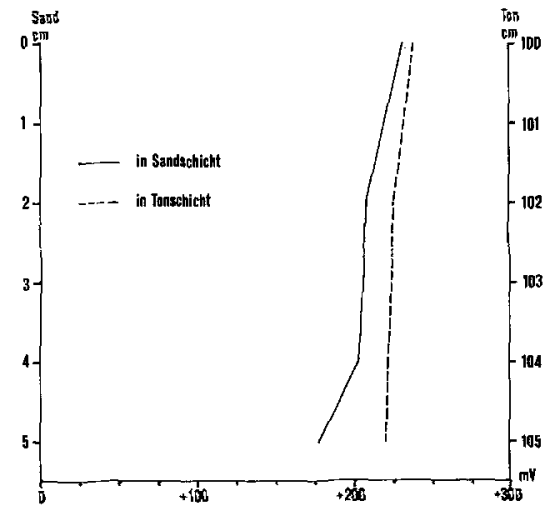

Abb. 6: Vertikaler Verlauf der Redoxpotentiale im Sammelabschnitt 3 im Sand und im Ton

Die ODR-Kurve für den Sammelabschnitt 1 verläuft ähnlich. Während an der Oberfläche ein Wert von 0,2 gemessen wurde, sinkt dieser bereits in $1 \mathrm{~cm}$ Tiefe unter den Grenzwert von 0,1. Der niedrigste ODR-Wert liegt bei 0,05 in $10 \mathrm{~cm}$ Tiefe. Die Wohnröhren dagegen weisen bis in $6 \mathrm{~cm}$ Tiefe noch Werte über 0,1 auf und heben sich somit auffallend deutlich vom umgebenden $\mathrm{O}_{2}$-freien Sediment ab. Parallel zu den Eh-Werten im Dezember 1973 lassen auch die ODR-Werte auf eine tiefergehende Oxidationsschicht in diesem Monat schließen (ODR 0,11 in $5 \mathrm{~cm}$ Tiefe).

Ganz andere Verhältnisse liegen im Sammelabschnitt 3 vor. Die Eh-Werte liegen durchweg über $+200 \mathrm{mV}$. Selbst die in ca. $1 \mathrm{~m}$ Bodentiefe beginnende, freigelegte Tonschicht weist nur positive Eh-Werte auf (Abb.6) und muß zur Oxidationszone gerechnet werden. In gleicher Weise läßt der Verlauf der ODR-Werte, die mit 0,17 ihren niedrigsten Stand erreichen, den Schluß auf eine gute Sauerstoffversorgung des Sedimentes zu (Abb. 7). 


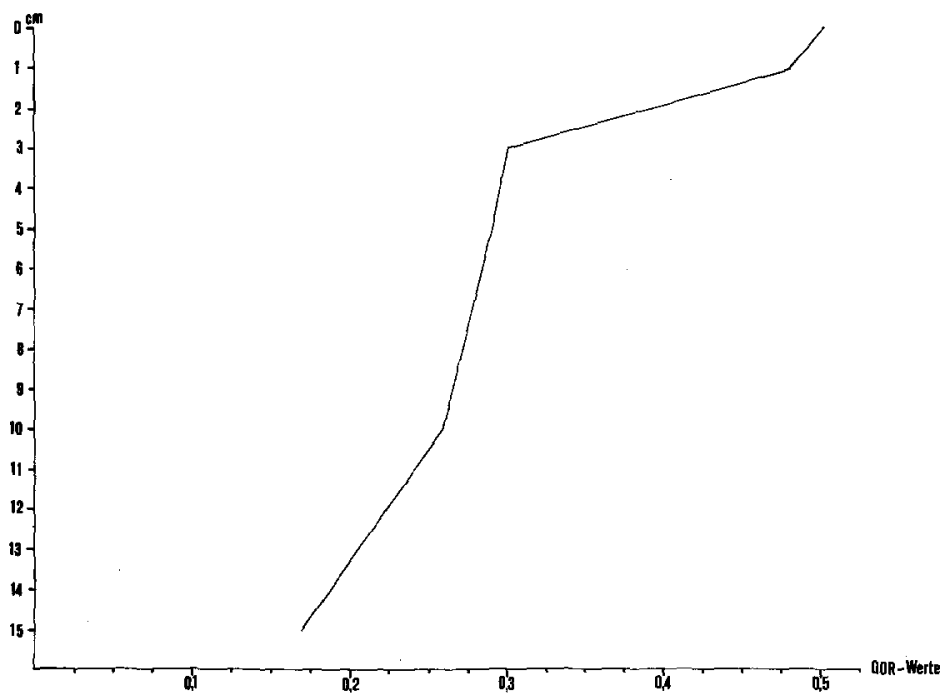

Abb. 7: Vertikaler Verlauf der ODR-Werte im Sammelabschnitt 3

\section{Korngrößenverteilung des Substrats}

Zur Bestimmung der Korngrößenklassen wurden etwa $100 \mathrm{~g}$ getrocknetes Sediment 15 Minuten lang maschinell gesiebt. Die Maschenweiten der hierbei verwendeten Siebe betrugen 2000, 1000,800,500, 250, 200, 125, 100 und $63 \mu \mathrm{m}$. Zur Charakterisierung der Korngrößen wurde die Korngrößeneinteilung nach Stripp (1969a) benutzt:

$$
\begin{aligned}
<63 \mu \mathrm{m} & \text { Schluff } \\
63-125 \mu \mathrm{m} & \text { sehr feiner Sand } \\
125-250 \mu \mathrm{m} & \text { Feinsand } \\
250-500 \mu \mathrm{m} & \text { Mittelsand } \\
500-1000 \mu \mathrm{m} & \text { Grobsand } \\
1000-2000 \mu \mathrm{m} & \text { sehr grober Sand } \\
>2000 \mu \mathrm{m} & \text { Kies }
\end{aligned}
$$

Die Sedimentzusammensetzung der Sammelabschnitte 1 und 3 ist den Säulendiagrammen der Abbildung 8 ( $a$ und $b$ ) zu entnehmen.

Die zur Beurteilung von Sedimenten wichtigen Kennzahlen, wie der Zentralwert oder Median MD, die Quartile $Q_{1}$ und $Q_{3}{ }^{*}$ (vgl. Schmidt, 1968) wurden aus der Zweipunkteform der Geradengleichung (Hartwig, 1973) errechnet:

Daraus ergibt sich:

$$
\frac{y-y_{1}}{x-x_{1}}=\frac{y_{2}-y_{1}}{x_{2}-x_{1}}
$$

$$
\mathrm{x}=\frac{\mathrm{y}\left(\mathrm{x}_{2}-\mathrm{x}_{1}\right)}{\mathrm{y}_{2}-\mathrm{y}_{1}}-\frac{\mathrm{y}_{1}\left(\mathrm{x}_{2}-\mathrm{x}_{1}\right)}{\mathrm{y}_{2}-\mathrm{y}_{1}}+\mathrm{x}_{1}
$$

* Median: derjenige Wert der Summenlinie, von dem aus $50 \%$ der Probe einen größeren und $50 \%$ einen kleineren Korndurchmesser aufweisen, Quartile $Q_{1}$ und $Q_{3}$ : grenzen in entsprechender Weise das obere und untere Viertel einer Probe ab (Schmidt, 1968). 

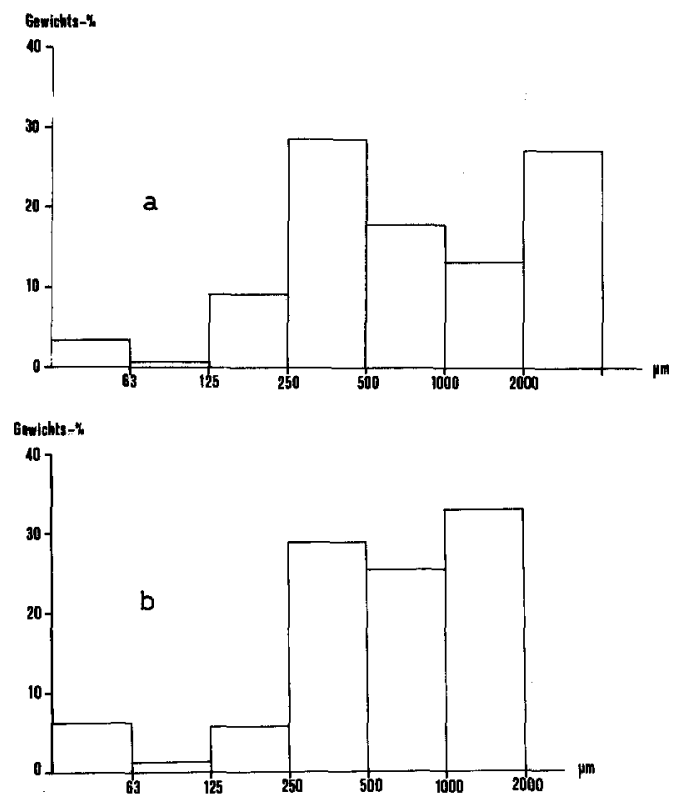

Abb. 8: Korngrößenverteilung im Sammelabschnitt 1(a) und Sammelabschnitt 3(b)

Weiterhin wurde der Sortierungskoeffizient $S_{0}$ aus den $Q$ uartilen $Q_{1}$ und $Q_{3}$ nach der Formel von Trask (1932) berechnet:

$$
\mathrm{S}_{\mathrm{o}}=\sqrt{\frac{\mathrm{Q}_{3}}{\mathrm{Q}_{1}}}
$$

Der Sortierungskoeffizient ist ein Maß für die Anzahl der am Sedimentaufbau beteiligten Korngrößenklassen. Bei einem völlig homogenen Sediment beträgt sein Wert 1. Je größer er wird, desto größer ist die Heterogenität des Sedimentes.

Die aufgeführten Kennzahlen sind in Tabelle 1 zusammengestellt.

Tabelle 1

Kennzahlen der Sedimente in den Sammelabschnitten 1 und 3

\begin{tabular}{|ccccc|}
\hline Sammelabschnitt & $\mathrm{MD}$ & $\mathrm{Q}_{1}$ & $\mathrm{Q}_{3}$ & $\mathrm{~S}_{\mathrm{o}}$ \\
\hline 1 & 733 & 355 & $>2000$ & $?$ \\
3 & 658 & 352 & $\mathbf{1 2 3 6}$ & 1,87 \\
\hline
\end{tabular}

Im Sammelabschnitt 1 besteht das Sediment zum überwiegenden Teil aus heterogenen Grobsänden (59\%), denen 37,7\% Feinsandanteile beigemischt sind. Die Substratzusammensetzung ist extrem heterogen. Der Sortierungskoeffizient war nicht zu berechnen.

Im Sammelabschnitt 3 dominieren mit $58 \%$ Gewichtsanteilen ebenfalls Grobsände. Feinsände sind zu 35,7\% vertreten. Bemerkenswert ist der relativ hohe Anteil an Schluff 
$(6,3 \%)$, der den visuell bereits verzeichneten Tonanteil bestätigt. Das Substrat weist mit einem $\mathrm{S}_{\mathrm{o}}$ - Wert von 1,87 einen hohen Heterogenitätsgrad auf.

\section{Detritusgehalt des Substrats}

Der Detritusgehalt (Gehalt an partikulärer organischer Substanz) wird außer von der Intensität der Wasserbewegung (Gerlach, 1953) auch vom mittleren Korndurchmesser des Sediments bestimmt (Dietrich et al., 1965). Er hängt weiterhin stark vom Grad der Umlagerung ab und kann an einer Probestelle je nach Witterungsbedingungen unterschiedliche Werte annehmen (Schmidt, 1968).

Der Gehalt an partikulärer organischer Substanz wurde durch Veraschen von Proben bei $550^{\circ} \mathrm{C}$ bestimmt. Der Glühverlust ist in Gewichtsprozenten des Trockensediments angegeben.

Das stark $\mathrm{H}_{2} \mathrm{~S}$-haltige Sediment im Sammelabschnitt 1 weist mit 2,47\% einen außerordentlich hohen Detritusgehalt auf. Erstaunlicherweise liegt er im Sammelabschnitt 3 mit 2,41\% nur geringfügig niedriger. Hierfür mag die Ähnlichkeit beider Sedimente bezüglich ihrer mittleren Korngröße von ausschlaggebender Bedeutung sein.

Der visuell nahezu steril erscheinende Sedimentstreifen um die "Lange Anna" im Sammelabschnitt 5 weist mit $1,4 \%$ einen erheblich niedrigeren Detritusgehalt auf.

\section{Temperaturen im Untersuchungsgebiet}

In Abbildung 9 sind die Kurven der Luftemperatur, der Temperatur des freien Wassers und die der Tümpel im Gezeitenbereich zusammengestellt. Den Temperaturangaben der Tümpel liegen eigene Messungen zugrunde, die durch Werte von Skaumal (1977) ergänzt wurden. Die Lufttemperaturdaten sowie die des freien Wassers wurden Angaben der Biologischen Anstalt Helgoland sowie des Deutschen Wetterdienstes entnommen.

Es zeigt sich, daß die Lufttemperatur und die Temperatur des freien Wassers sich erwartungsgemäß gleichsinnig verhalten, wobei die Wassertemperaturkurve gegenüber der der Lufttemperatur zeitlich um etwa einen Monat versetzt ist. Im Gezeitenbereich dagegen läßt die Temperaturkurve der flachen stehenbleibenden Tümpel in viel stärkerem Maße eine direkte Abhängigkeit von der Lufttemperatur erkennen.

\section{Salinität}

Die Salzgehaltsschwankungen des freien Meereswassers bei Helgoland hielten sich während des Untersuchungszeitraumes mit minimal 29,60\% im April 1973 und maximal 33,35\% im März 1974 in den üblichen Grenzen.

Größere Salzgehaltsschwankungen können im Tidenbereich auftreten. Sie steigen mit zunehmender Entfernung von der Wasserlinie. Durch Regenfälle kommt es während der Niedrigwasserperioden zur Aussüßung der oberen Sedimentschichten; andererseits bedingen hohe Temperaturen, die mit einer hohen Verdunstungsrate einhergehen, einen Anstieg des Salzgehaltes (Gerlach, 1953; Hartwig, 1973). 


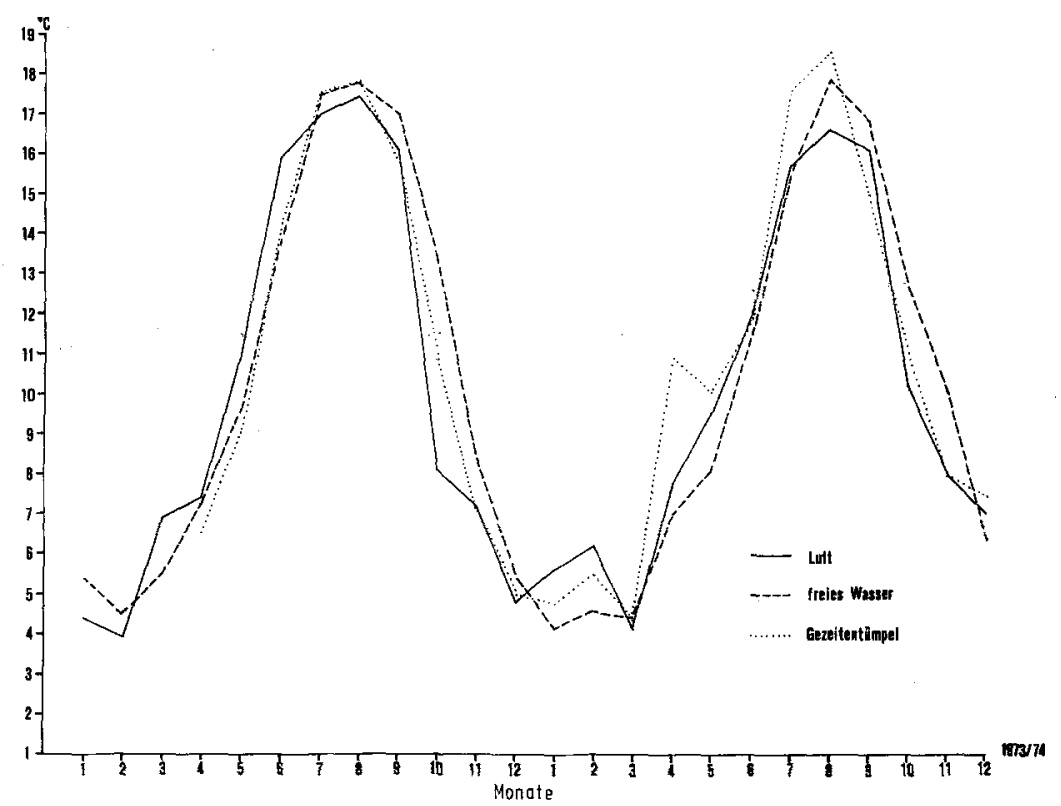

Abb. 9: Monatliche Mittelwerte der Luft- und Wassertemperaturen (freies Wasser und Gezeitentümpel) im Jahresverlauf (nach Daten der Biologischen Anstalt Helgoland)

Für die speziell im Gezeitenbereich angesiedelten Organismen bedeutet dies, daß sie mit spezifischen Anpassungsmechanismen an stark wechselnde Salzgehalte ausgestattet sein müssen.

\section{Verteilung der Polychaeten im Helgoländer Felslitoral}

53 Polychaetenarten aus 18 Familien wurden während des Untersuchungszeitraumes im Helgoländer Felslitoral gefunden (vgl. Tab, 2).

\section{Horizontalverteilung}

In Tabelle 3 ist die horizontale Verteilung der gefundenen Polychaetenarten, bezogen auf die einzelnen Sammelabschnitte, dargestellt. Die Anordnung der Arten erfolgte gemäß ihrer absoluten Häufigkeit in den von Osten nach Westen aufeinanderfolgenden Sammelabschnitten. Somit stehen Arten mit einem Häufigkeitsmaximum im Abschnitt 1 bzw, vor 1 (Nereis virens, Nereis succinea) ganz oben auf der Liste, während Arten, die ihr Häufigkeitsmaximum im Abschnitt 5 erreichen, als letzte aufgeführt sind. Die Häufigkeitsangaben erfolgten in absoluten Individuenzahlen.

Der sich östlich an das Untersuchungsgebiet anschließende Sandstrand v o r Sammelabschnitt 1 ist durch das regelmäßige Vorkommen von Nereis virens charakterisiert. Nereis succinea wurde ausschließlich hier, wenn auch nur in geringer Anzahl, gefunden. Im 
Tabelle 2

Im Helgoländer Felslitoral nachgewiesene Polychaetenarten

Lepidonotus squamatus (Linné, 1767)

Harmothoë impar (Johnston, 1839)

Gattyana cirrosa (Pallas, 1766)

Pholoë minuta (Fabricius, 1870)

Sthenelais boa (Johnston, 1839)

Eteone longa (Fabricius, 1780)

Anaitides maculata (Linné, 1767)

Eumidia punctifera (Grube, 1860)

Eumidia sanguinea (Oersted, 1843)

Eulalia viridis (Linné, 1767)

Kefersteinia cirrata (Keferstein, 1862)

Micropbthalmus sczelkowii Mecznikow, 1865

Syllis gracilis Grube, 1840

Typosyllis byalina (Grube, 1863)

Typosyllis armillaris (O. F. Müller, 1776)

Syllides articulocirratus n. $\mathrm{sp}$.

Brania cf. pusilla (Dujardin, 1839)

Sphaerosyllis bystrix Claparéde, 1863

Exogone naidina Oersted, 1845

Autolytus prolifer (O. F. Müller, 1776)

Autolytus edwarsi Saint-Joseph, 1887

Nereis pelagica Linné, 1761

Nereis succinea Frey \& Leuckart, 1847

Nereis virens Sars, 1835

Sphaerodorum flavum Oersted, 1843

Ophryatrocha gracilis Huth, 1934

Polydora ligni Webster, 1879
Polydora ciliata (Johnston, 1838)

Pygospio elegans Claparéde, 1863

Scolelepis squamata (Müller, 1789)

Malacoceros vulgaris (Johnston, 1827)

Malacoceros fuliginosus (Claparéde, 1868)

Caulleriella bioculata parva $\mathrm{n}$. ssp.

Caulleriella caputesocis (Saint-Joseph, 1894)

Tharyx multibranchits (Grube, 1863)

Dodecaceria concharum Oersted, 1843

Zeppelina monostyla (Zeppelin, 1883)

Zeppelina mediopigmentata n. sp.

Flabelligera affinis (M. Sars, 1829)

Pherusa flabellata (Sars, 1872)

Pherusa plumosa (O. F. Müller, 1776)

Capitella capitata (Fabricius, 1780)

Capitomastus giardi (Mesnil, 1897)

Arenicola marina (Linné, 1758)

Amphitrite figulus (Dalyell, 1853)

Nicolea zostericola (Oersted, 1844)

Lanice conchilega (Pallas, 1766)

Sabellaria spinulosa Leuckart, 1849

Fabricia sabella (Ehrenberg, 1836)

Pomatoceros triqueter (Linné, 1758)

Janua pagenstecheri (Quatrefages, 1865)

Spirorbis tridentatus (Levinsen, 1883)

Spirorbis spirorbis (Linné, 1858)

östlichen Teil trat außerdem Nereis pelagica relativ häufig auf, während die Häufigkeit dieser Art - wie auch die von Nereis virens - in Richtung auf den Abschnitt 1 hin immer mehr zurückging und statt dessen eine Zunahme von Malacoceros fuliginosus und Capitella capitata $\mathrm{zu}$ verzeichnen war, die mit einem zunehmenden $\mathrm{H}_{2} \mathrm{~S}$-Gehalt des Substrats einherging.

Im S a m me labs chnit 1 dominieren die Arten Malacoceros fuliginosus und Capitella capitata, die vorzugsweise im $\mathrm{H}_{2} \mathrm{~S}$-haltigen Grobsediment vorkamen. Capitella capitata erreichte in diesem Abschnitt ihr Häufigkeitsmaximum, ebenso wie Arenicola marina, die aus anderen Abschnitten nur in Einzelfunden vorliegt. Neben den genannten Arten ist das regelmäßige Auftreten der nur wenige Millimeter großen Art Micropbthalmus sczelkowii erwähnenswert, die als Element der Sandlückenfauna stets zusammen mit Malacoceros fuliginosus und Capitella capitata gefunden wurde. Sie ist bisher aus schlickigsandigen Gebieten der Nordseewatten, hauptsächlich von Sylt, bekannt (Westheide, 1966, 1967, 1972), lebt auch in detritusreichen Schillansammlungen, wurde bisher jedoch nicht in $\mathrm{H}_{2} \mathrm{~S}$-haltigem Milieu nachgewiesen.

$\mathrm{S}$ a m m e la b s ch n it t 2 unterscheidet sich - wie bereits beschrieben - im Aufbau kaum vom ersten Abschnitt. Erwartungsgemäß gleicht demzufolge auch die Faunenzusammensetzung in diesem Abschnitt der von Abschnitt 1. Es dominieren wiederum die beiden Arten Malacoceros fuliginosus und Capitella capitata, wobei bei Capitella capitata aller- 
Tabelle 3

Horizontalverteilung der Polychaetenarten inn Helgoländer Felslitoral

\begin{tabular}{|c|c|c|c|c|c|c|c|}
\hline $\begin{array}{ll} & \text { Sammelabschnitt } \\
\text { Species } & \end{array}$ & vor 1 & 1 & 2 & 3 & 4 & 5 & Summe \\
\hline \multirow{3}{*}{$\begin{array}{l}\text { Nereis virens } \\
\text { Nereis succinea } \\
\text { Capitella capitata } \\
\text { Malacoceros fuliginosus } \\
\text { Arenicola marina }\end{array}$} & • & 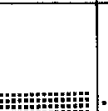 & $\cdot$ & $\cdots$ & \multirow[b]{3}{*}{ • } & \multirow[b]{3}{*}{ 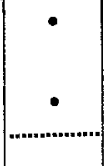 } & 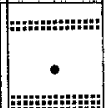 \\
\hline & & 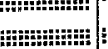 & 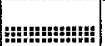 & 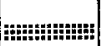 & & & | \\
\hline & - & $\ldots$ & $\cdot$ & $\cdot$ & & & , \\
\hline $\begin{array}{l}\text { Polydora ligni } \\
\text { Zeppelina mediopigmentata } \\
\text { Caulleriella caputesocis }\end{array}$ & & - & - & $\bullet$ & & - & : \\
\hline $\begin{array}{l}\text { Gattyana cirrosa } \\
\text { Eumidia sanguinea }\end{array}$ & - & - & - & $\bullet$ & $\dot{\bullet}$ & - & \\
\hline $\begin{array}{l}\text { Lanice conchilega } \\
\text { Scolelepis squamata } \\
\text { Pherusa flabellata } \\
\text { Flabelligera affinis } \\
\text { Zeppelina monostyla }\end{array}$ & $\bullet$ & - & - & :………" & $\begin{array}{l}\bullet \\
\bullet \\
\bullet\end{array}$ & $\cdot$ & 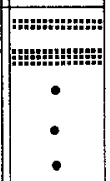 \\
\hline $\begin{array}{l}\text { Exogone naidina } \\
\text { Eumidia punctifera } \\
\text { Malacoceros vulgaris } \\
\text { Typosyllis hyalina } \\
\text { Brania cf. pusilla }\end{array}$ & & - & & - & $\begin{array}{l}\dot{ } \\
\dot{ } \\
\dot{ }\end{array}$ & - & $\dot{\bullet}$ \\
\hline $\begin{array}{l}\text { Caulleriella bioculata parva } \\
\text { Syllis gracilis } \\
\text { Micropbthalmus sczelkowoii }\end{array}$ & - & & • & $\ddot{\cdot}$ & 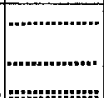 & $\because$ & 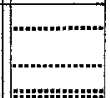 \\
\hline $\begin{array}{l}\text { Eteone longa } \\
\text { Sphaerodorum flarum }\end{array}$ & & & & & & : & $\mid$ \\
\hline $\begin{array}{l}\text { Pherusa plumosa } \\
\text { Amphitrite figulus } \\
\text { Syllides articulocirratus } \\
\text { Dodecaceria concharum } \\
\text { Kefersteinia cirrata }\end{array}$ & - & & & & - & $\dot{:}$ & $\begin{array}{l}\dot{ } \\
\dot{.} \\
\dot{.}\end{array}$ \\
\hline $\begin{array}{l}\text { Pholoë minuta } \\
\text { Harmothoë impar } \\
\text { Autolytus edwarsi } \\
\text { Nicolea zostericola } \\
\text { Sthenelais boa }\end{array}$ & : & & - & $\bullet$ & $\begin{array}{l}\dot{ } \\
\dot{ } \\
\dot{ }\end{array}$ & \begin{tabular}{c||}
$\ddot{0}$ \\
$\ddot{0}$ \\
$\cdots \cdots$ \\
$\cdots \cdots$
\end{tabular} & 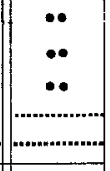 \\
\hline $\begin{array}{l}\text { Sphaerosyllis bystrix } \\
\text { Tharyx multibranchiis } \\
\text { Sabellaria stinulosa }\end{array}$ & & $\bullet$ & & $\bullet$ & - & $\cdots \cdots$ & \\
\hline $\begin{array}{l}\text { Sabellaria spinulosa } \\
\text { Lepidonotus squamatus } \\
\text { Eulalia viridis }\end{array}$ & $\ddot{\bullet}$ & •• & & - & $\ldots \cdots \cdots \cdots \cdots$ & $\ldots \cdots \cdots \cdots$ & 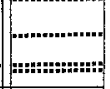 \\
\hline $\begin{array}{l}\text { Autolytus prolifer } \\
\text { Anaitides maculata }\end{array}$ & $\ddot{\bullet}$ & $\bullet$ & $\bullet$ & & & & | \\
\hline $\begin{array}{l}\text { Typosyllis armillaris } \\
\text { Pomatoceros triqueter } \\
\text { Ophryotrocha gracilis }\end{array}$ & & - & 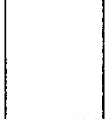 & $\stackrel{\bullet}{\bullet}$ & 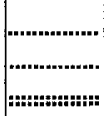 & |: & : \\
\hline Capitomastus giardi & & & $\ldots$ & 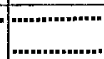 & 年 & |: & 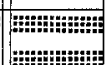 \\
\hline $\begin{array}{l}\text { Nereis pelagica } \\
\text { Janua pagenstecheri } \\
\text { Spirorbis tridentatus } \\
\text { Pygospio elegans }\end{array}$ & - & & & & : & : & 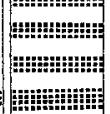 \\
\hline $\begin{array}{l}\text { Polydora ciliata } \\
\text { Spirorbis spirorbis } \\
\text { Fabricia sabella }\end{array}$ & • & | & 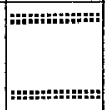 & : & 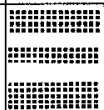 & 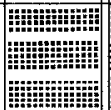 & $\frac{1}{3}$ \\
\hline $\begin{array}{ll}. & \text { unter } 5 \\
\cdots & 5-10 \\
\cdots & 10-50\end{array}$ & 25 & & & 㐩 & $\begin{array}{l}500-1000 \\
\text { iher } 1000\end{array}$ & & \\
\hline
\end{tabular}


dings ein deutlicher zahlenmäßiger Rückgang gegenüber Abschnitt $1 \mathrm{zu}$ verzeichnen ist. Als einzige Art erreicht Polydora ligni in diesem Abschnitt ihre maximale Häufigkeit.

Im S a m m labschnitt 3 ist eine Dominanz von Scolelepis squamata zu beobachten. Diese Art hat in diesem Abschnitt zugleich ihr Häufigkeitsmaximum. Das gilt in gleicher Weise für Gattyana cirrosa, Eumidia sanguinea, Malacoceros fuliginosus und Lanice conchilega.

Im $\mathrm{S}$ a m m e la b s h n it t 4 ist Fabricia sabella dominant, gefolgt von Polydora ciliata, Pygospio elegans und Spirorbis spirorbis. Keine der genannten Arten weist allerdings in diesem Abschnitt ihr Häufigkeitsmaximum auf. Dies ist der Fall bei Eumidia punctifera, Microphthalmus sczelkowii, Syllis gracilis, Typosyllis byalina, Brania cf. pusilla, Malacoceros vulgaris und Caulleriella bioculata parva.

Im $S$ a $m$ m e la b s c h n it t 5 haben die weitaus meisten Arten ihr Häufigkeitsmaximum. Es handelt sich hierbei um Lepidonotus squamatus, Harmothoë impar, Pholö̈ minuta, Sthenelais boa, Anaitides maculata, Eulalia viridis, Kefersteinia cirrata, Typosyllis armillaris, Syllides articulocirratus, Sphaerosyllis bystrix, Autolytus prolifer, Autolytus edwarsi, Nereis pelagica, Ophryotrocha gracilis, Polydora ciliata, Pygospio elegans, Tharyx multibranchiis, Dodecaceria concharum, Capitomastus giardi, Nicolea zostericola, Sabellaria spinulosa, Fabricia sabella, Pomatoceros triqueter, Janua pagenstecheri, Spirorbis tridentatus und Spirorbis spirorbis. Es dominiert Fabricia sabella, gefolgt von Spirorbis spirorbis und Polydora ciliata.

Für eine Reihe von Arten, die nur ein- oder zweimal gefunden wurden, ist es wenig sinnvoll, Häufigkeitsverteilungen anzugeben. Diese Arten sind an ihren entsprechenden Fundorten in Tabelle 3 aufgeführt, jedoch auf Grund ihrer zu geringen Individuenzahl bei den Angaben zur Horizontalverteilung unberücksichtigt geblieben. Es handelt sich hierbei um die Arten Exogone naidina, Sphaerodorum flavum, Caulleriella caputesocis, Zeppelina monostyla, Zeppelina mediopigmentata, Flabelligera affinis, Pherusa plumosa und Ampbitrite figulus.

Die in Tabelle 3 dargestellte Horizontalverteilung läßt über die Aussagen zu den einzelnen Polychaetenarten hinaus drei allgemeine, gleichlaufende Tendenzen erkennen: (1) Die Zahl der Arten nimmt vom Sammelabschnitt 1 bis zum Sammelabschnitt 5 - von einer Ausnahme abgesehen - kontinuierlich zu. (2) In gleicher Weise ist eine Steigerung der Artenzahlen, die ihr Häufigkeitsmaximum in den jeweiligen Sammelabschnitten haben, von Abschnitt 1 nach $5 \mathrm{zu}$ verzeichnen. (3) Die Tendenz einzelner Arten, in höheren Individuenzahlen aufzutreten, ist zumindest in den Abschnitten 3 bis 5 zu erkennen.

$\mathrm{Zu}$ 1: In Tabelle 4 sind unter den Sammelabschnitten 1 bis 5 die dort jeweils gefundenen Artenzahlen ( $\mathrm{n}$ ) aufgeführt sowie der entsprechende Prozentsatz, bezogen auf die Gesamtzahl von 53 gefundenen Arten. Das geringfügige Absinken der Artenzahl im Sammelabschnitt 2 gegenüber dem Abschnitt 1 läßt sich damit erklären, daß im Sammelabschnitt 1 angespülte Laminaria-Rhizoide gesammelt und ausgewertet wurden, die im Abschnitt 2 vollkommen fehlten. Die Zahlen der übrigen Abschnitte lassen die oben erläuterte Tendenz deutlich erkennen.

$\mathrm{Zu}$ 2: Aus Tabelle 4 geht ebenfalls die Verteilung der Häufigkeitsmaxima (A) auf die einzelnen Sammelabschnitte hervor. Für den geringfügigen Abfall im Abschnitt 2 gegenüber Abschnitt 1 ist oben bereits eine Erläuterung gegeben worden, die sinngemäß hier ebenfalls gilt. 
Zu 3: Zur Veranschaulichung dieser Aussage sind in Tabelle 5 exemplarisch die maximalen Individuenzahlen $(\mathrm{n})$ der in den einzelnen Sammelabschnitten jeweils dominierenden Arten zusammengestellt.

T'abelle 4

Horizontale Verteilung der gefundenen Gesamt-Artenzahl (n), der Anzahl Arten, die im jeweiligen Sammelabschnitt ihre höchsten Individuenzahlen aufweisen (A) sowie der entsprechenden Prozentwerte $(53$ Arten $\hat{=} 100 \%)$

\begin{tabular}{|cccccc|}
\hline Sammelabschnitt & 1 & 2 & 3 & 4 & 5 \\
\hline $\mathrm{n}$ & 17 & 16 & 27 & 39 & 41 \\
$\%$ & 32,1 & 30,2 & 50,9 & 73,6 & 77,4 \\
$\mathrm{~A}$ & 2 & 1 & 5 & 7 & 26 \\
$\%$ & 3,8 & 1,9 & 9,4 & 13,2 & 49,1 \\
\hline
\end{tabular}

Tabelle 5

Horizontale Verteilung der maximalen Individuenzahlen der in den Sammelabschnitten jeweils dominierenden Polychaetenarten im Helgoländer Felslitoral

\begin{tabular}{|ccl|}
\hline Sammelabschnitt & $\mathrm{n}$ & \multicolumn{1}{c|}{ Species } \\
\hline 1 & 306 & Capitella capitata \\
2 & 117 & Malacoceros fuliginosus \\
3 & 226 & Scolelepis squamata \\
4 & 531 & Fabricia sabella \\
5 & 802 & Fabricia sabella \\
\hline
\end{tabular}

\section{Vertikalverteilung}

Abbildung 10 und Tabelle 6 zeigen die allgemeine vertikale Verteilung der Polychaetenarten im Helgoländer Felslitoral sowie in den einzelnen Sammelabschnitten. Dabei wurden die Zonen des Eulitorals - ohne das Supralitoral - sowie der obere Sublitoralsaum berücksichtigt. Das tiefer gelegene Sublitoral blieb bei der Beurteilung der Vertikalverteilung in den einzelnen Sammelabschnitten unberücksichtigt, da dieser Bereich wegen der nur ausnahmsweise möglichen Begehbarkeit in den einzelnen Abschnitten nicht repräsentativ besammelt werden konnte und mithin die geringe Probenzahl nicht ausreicht, um gesicherte Aussagen zu machen.

Das tiefer gelegene Sublitoral wurde jedoch in die allgemeine Auswertung der vertikalen Verteilung mit einbezogen, da einzelne Polychaetenarten, die ausschließlich in Laminaria-Rhizoiden gefunden wurden, sehr wohl eine Aussage über ihre vertikale Verbreitung zulassen, ohne daß sie mit Sicherheit einem bestimmten Sammelabschnitt zugeordnet werden könnten. 
Vertikalverteilung in den einzelnen Sammelabschnitten

Der östlich vor dem Sammelabschnitt 1 gelegene Strandstreifen wird im oberen Eulitoral von 5 Arten bewohnt; im mittleren Eulitoral sind es 10. Unter ihnen ist Nereis virens dominant. Im unteren Eulitoral sinkt die Zahl der Arten auf $2 \mathrm{ab}$ (Nereis virens und Lanice conchilega). Im oberen Sublitoral schließlich erreicht die Artenzahl mit 14 ihr Maximum.

In der Tendenz ähnlich verhält sich die Polychaetenfauna im S a mmelabschnitt 1. Hier ist ebenfalls im oberen Sublitoral die größte Artenzahl (8) festzustellen. Im oberen und mittleren Eulitoral bleibt die Artenzahl mit 7 bzw. 6 etwa konstant (es dominieren hier die Arten Capitella capitata und Malacoceros fuliginosus), während im unteren Eulitoral die geringste Artendichte (2) beobachtet wird.

Das sprunghafte, ungewöhnliche Absinken der Artenzahlen im unteren Eulitoral, sowohl am Strand vor Abschnitt 1 als auch im Sammelabschnitt 1 selbst, hat möglicherweise seine Ursache in einer strömungsbedingten, besonders kräftigen Wellenbewegung in dieser Zone, die eine ständige starke Substratumlagerung zur Folge hat. Durch diese wiederum kann die Ansiedlung vor allem empfindlicherer Organismen erschwert, wenn nicht sogar verhindert werden, zumal auch kein Algenbewuchs in diesem Bereich angetroffen wird.

Im $S$ a m m e la b s ch n it t 2 steigt die Artenzahl von 4 im oberen Eulitoral auf 11 im mittleren Eulitoral an, wobei die Arten Malacoceros fuliginosus und Capitella capitata die größte Individuendichte stellten. Das anschließende Absinken auf 6 (im unteren Eulitoral) bzw. 5 Arten (im oberen Sublitoral) muß als untypisch angesehen werden. Die Artenzahlen würden in diesen Zonen mit großer Wahrscheinlichkeit höher liegen, wenn die Wasserstandsverhältnisse eine ausgedehntere Sammeltätigkeit in diesen Bereichen gestattet hätten. Da jedoch im Gegensatz zu den Sammelabschnitten 4 und 5 im Abschnitt 2 nur ausnahmsweise ein sehr kleiner Teil der unteren Zonen trockenfiel, läßt das vorliegende Polychaetenmaterial für diesen Abschnitt keine gesicherten Aussagen über die vertikale Verbreitung der Arten zu.

Im Sammelabschnitt 3 ist eine deutliche Zunahme der Gesamtartenzahl gegenüber den Abschnitten 1 und 2 zu verzeichnen. Das obere Eulitoral wird von 8 Arten bewohnt, unter denen Scolelepis squamata mit Abstand die höchste Individuendichte erreicht. Malacoceros fuliginosus und Capitella capitata, die dominierenden Arten der beiden ersten Sammelabschnitte, sind in dieser Zone nur in Einzelexemplaren vertreten. Ihr Häufigkeitsmaximum im Abschnitt 3 verschiebt sich vom oberen Eulitoral ins mittlere und untere Eulitoral, während der ihnen in den Abschnitten 1 und 2 eigene Lebensraum im Abschnitt 3 von Scolelepis squamata eingenommen wird.

Die mögliche Ursache dieser Verdrängung wird später noch diskutiert werden. Im mittleren Eulitoral erreicht die Artenanzahl mit 16 Arten ihr Maximum. Der anschlieBende, geringfügige Abfall auf 13 bzw. 14 Arten im unteren Eulitoral bzw. oberen Sublitoral ist zu vernachlässigen. Für diesen gilt - wenn auch in erheblich abgeschwächtem Maße - die für den Abschnitt 2 für das gleiche Phänomen gegebene Erläuterung.

$\mathrm{S}$ a m m e labschn it t 4 läßt vom oberen Eulitoral zum oberen Sublitoral hin eine stufenweise Erhöhung der Artenzahlen erkennen. Während sie im oberen Eulitoral 10 
beträgt, steigt sie über $20 \mathrm{im}$ mittleren Eulitoral auf $23 \mathrm{im}$ unteren Eulitoral und erreicht im oberen Sublitoral mit 24 ihr Maximum.

In gleicher Weise stellt sich die Vertikalverteilung im $\mathrm{S}$ a $\mathrm{m}$ m e la b s ch n i t 5 dar. Die maximale Artenzahl wird mit 26 im oberen Sublitoral erreicht. Landwärts sinkt sie kontinuierlich über 25 im unteren Eulitoral und 23 im mittleren Eulitoral auf 15 im oberen Eulitoral. In den Abschnitten 4 und 5 ist wiederum eine Erhöhung der Gesamtartenzahl gegenüber den vorangegangenen Abschnitten festzustellen.

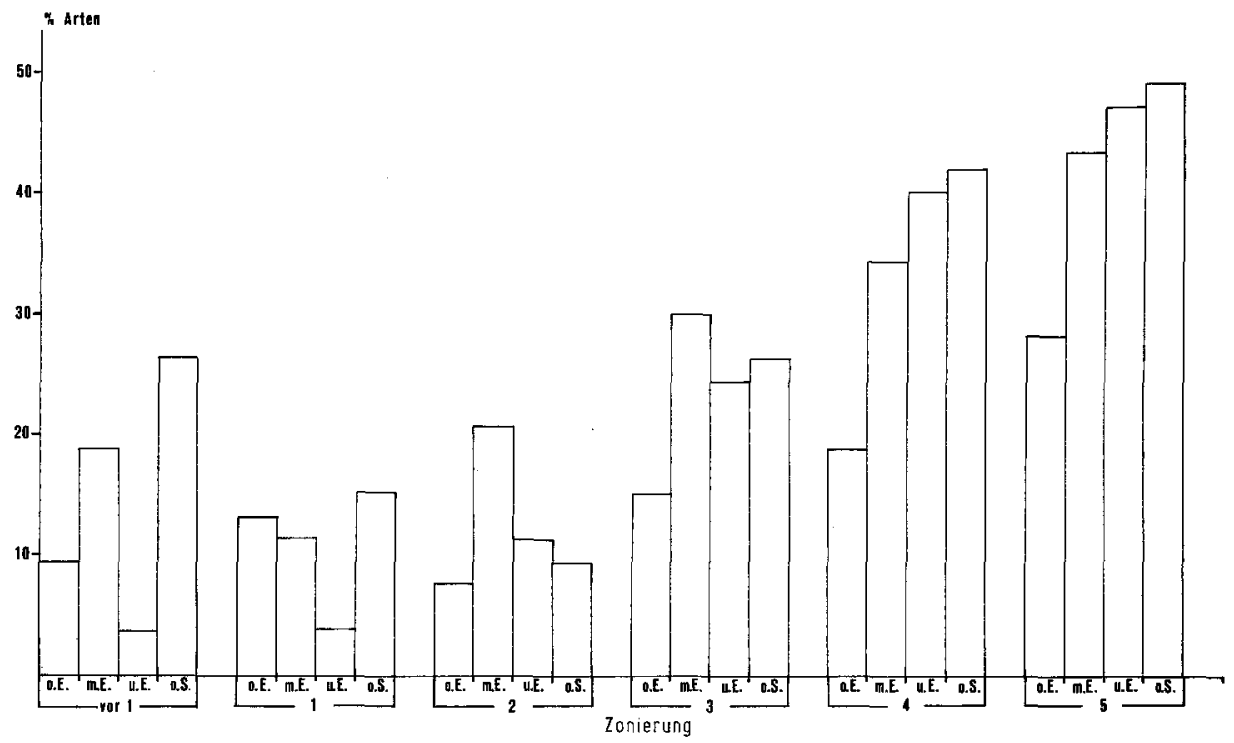

Abb. 10: Vertikalverteilung der Polychaetenfauna in den einzelnen Sammelabschnitten im Helgoländer Felslitoral; o. E. oberes Eulitoral; m. E. mittleres Eulitoral; u. E. unteres Eulitoral; o. S. oberes Sublitoral

Zur besseren Information sind die in Abbildung 10 in Prozenten der Gesamtartenzahl gemachten Angaben bei der vorstehenden Erläuterung des Diagramms als absolute Zahlenwerte angegeben.

Zusammenfassend sind folgende Fakten festzuhalten: (1) Die Artenzahl steigt abgesehen von wenigen Ausnahmen, die erläutert werden - in allen Sammelabschnitten mit zunehmender Tiefe an. (2) Die Zunahme der Artenzahl geht einher mit einer länger anhaltenden Wasserbedeckung des betreffenden Areals. (3) Das Säulendiagramm (Abb. 10) zeigt - wie bereits aus der Horizontalverteilung ersichtlich -, daß die Gesamtzahl der Arten vom Sammelabschnitt 1 zum Sammelabschnitt 5 hin kontinuierlich zunimmt.

\section{Vertikalverteilung im Gesamtuntersuchungsraum}

Die vertikale Verteilung der Polychaetenfauna im gesamten untersuchten Helgoländer Felslitoral wird aus Tabelle 6 ersichtlich. Neben den Arten sind die Individuenzahlen in den einzelnen Zonierungen in Prozenten der Gesamtindividuenzahl der betreffenden Art 
Tabelle 6

Vertikale Verteilung der Polychaeten im Helgoländer Felslitoral

\begin{tabular}{|c|c|c|c|c|c|}
\hline Species Zonierung & $\begin{array}{l}\text { Oberes } \\
\text { Eulitoral }\end{array}$ & $\begin{array}{l}\text { Mittleres } \\
\text { Eulitoral }\end{array}$ & $\begin{array}{l}\text { Unteres } \\
\text { Eulitoral }\end{array}$ & $\begin{array}{l}\text { Oberes } \\
\text { Sublitoral }\end{array}$ & $\begin{array}{l}\text { Mittleres } \\
\text { Sublitoral }\end{array}$ \\
\hline $\begin{array}{l}\text { Scolelepis squamata } \\
\text { Micropbthalmus sczelkowii } \\
\text { Ophryotrocha gracilis } \\
\text { Pygospio elegans } \\
\text { Arenicola marina }\end{array}$ & 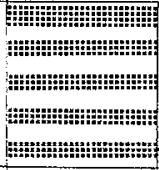 & 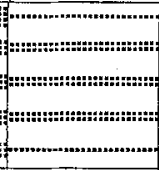 & $\bullet$ & - & - \\
\hline $\begin{array}{l}\text { Eumidia sanguinea } \\
* \text { Exogone naidina } \\
\text { Lanice conchilega } \\
\text { Spirorbis spirorbis } \\
\text { Polydora ligni }\end{array}$ & $\bullet$ & 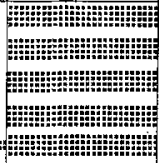 & 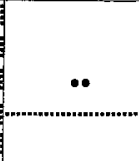 & - & \\
\hline $\begin{array}{l}\text { Janua pagenstecheri } \\
\text { Nereis succinea } \\
\text { Nereis virens } \\
\text { Capitella capitata } \\
\text { Malacoceros fuliginosus }\end{array}$ & 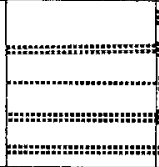 & | & $\begin{array}{c}\mid \\
\bullet \\
\bullet\end{array}$ & $=$ & \\
\hline $\begin{array}{l}\text { Polydora ciliata } \\
\text { Capitomastus giardi } \\
\text { Fabricia sabella } \\
\text { Pomatoceros triqueter } \\
\text { Harmothoë impar }\end{array}$ & $\begin{array}{c}\bullet \\
\bullet\end{array}$ & 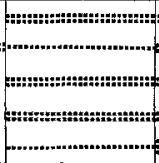 & 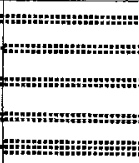 & (1) & $\left\{\begin{array}{c}\bullet \\
\bullet \\
\bullet \\
\bullet \\
\ldots \ldots \ldots \ldots \ldots\end{array}\right.$ \\
\hline $\begin{array}{l}\text { Brania cf. pusilla } \\
\text { Sphaerosyllis bystrix } \\
\text { Kefersteinia cirrata } \\
\text { Sabellaria spinulosa } \\
\text { Tharyx multibranchiis }\end{array}$ & 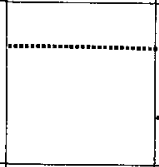 & $\bullet$ & 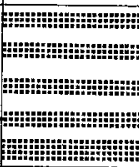 & (1) & 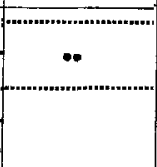 \\
\hline $\begin{array}{l}\text { Syllides articulocirratus } \\
\text { Dodecaceria concharum } \\
\text { * Malacoceros vulgaris } \\
\text { * Flabelligera affinis } \\
\text { Spirorbis tridentatus }\end{array}$ & $\bullet$ & 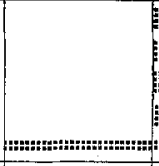 &  & 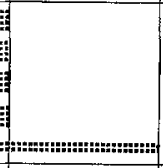 & \\
\hline $\begin{array}{l}\text { Syllis gracilis } \\
\text { Gattyana cirrosa } \\
\text { Anaitides maculata } \\
\text { * Zeppelina mediopigmentata } \\
\text { Nereis pelagica }\end{array}$ & 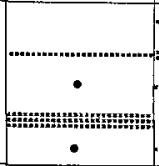 & | & (2) & 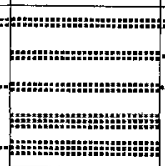 & 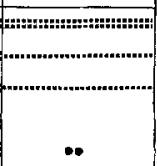 \\
\hline $\begin{array}{l}\text { Lepidonotus squamatus } \\
\text { Autolytus prolifer } \\
\text { Stbenelais boa } \\
\text { Eumidia punctifera } \\
\text { Typosyllis armillaris }\end{array}$ & 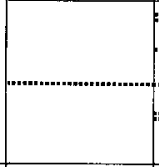 & 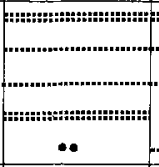 & 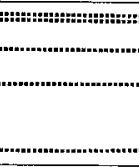 & 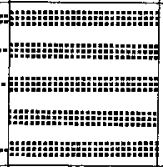 & $\bullet$ \\
\hline $\begin{array}{l}\text { Eulalia viridis } \\
\text { Autolytus edwarsi } \\
\text { Amphitrite figulus } \\
\text { Caulleriella caputesocis } \\
\text { * Eteone longa }\end{array}$ & & - & (1) & 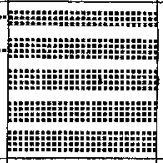 & - \\
\hline $\begin{array}{l}\text { * Zeppelina monostyla } \\
\text { * Pherusa flabellata } \\
\text { * Pherusa plumosa } \\
\text { Nicolea zostericola } \\
\text { Pboloë minuta }\end{array}$ & & ๑ & & : & 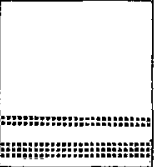 \\
\hline $\begin{array}{l}\text { Typosyllis byalina } \\
\text { Caulleriella bioculata parva } \\
\text { * Sphaerodorum flawum }\end{array}$ & & & & $\cdots$ & 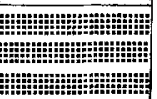 \\
\hline
\end{tabular}


aufgeführt. Arten, die so selten gefunden wurden, daß nur unsichere Aussagen über ihre vertikale Verbreitung gemacht werden können, sind in Tabelle 6 mit einem Stern (*) versehen. Folgende Aussagen lassen sich aus Tabelle 6 ablesen: (1) Die Zahl der Arten nimmt rom Eulitoral zum Sublitoral hin zu. (2) Die Zahl der ein Häufigkeitsmaximum aufweisenden Arten steigt ebenfalls vom Eulitoral zum Sublitoral hin an.

In Tabelle 7 sind die Artenzahlen (n) der jeweiligen Zonierung sowohl in absoluten Werten als auch in Prozenten der Gesamtartenzahl zusammengestellt. Die Angaben über die Artenzahl mit Häufigkeitsmaxima (A) in den einzelnen Zonierungen sind ebenfalls der Tabelle 7 zu entnehmen.

Tabelle 7

Vertikale Verteilung der gefundenen Gesamt-Artenzahl (n), der Anzahl der Arten, die in der jeweiligen Zone ihre höchsten Individuenzahlen aufweisen (A) sowie der entsprechenden Prozentwerte $(53$ Arten $\hat{=} 100 \%)$

\begin{tabular}{|cccccc|}
\hline Zonierung & $\begin{array}{c}\text { oberes } \\
\text { Eulitoral }\end{array}$ & $\begin{array}{c}\text { mittleres } \\
\text { Eulitoral }\end{array}$ & $\begin{array}{c}\text { unteres } \\
\text { Eulitoral }\end{array}$ & $\begin{array}{c}\text { oberes } \\
\text { Sublitoral }\end{array}$ & $\begin{array}{c}\text { (mittleres } \\
\text { Sublitoral) }\end{array}$ \\
\hline $\mathrm{n}$ & 22 & 35 & 34 & 35 & $(20)$ \\
$\%$ & 41,5 & 66,0 & 64,2 & 66,0 & $(37,7)$ \\
$\mathrm{A}$ & 5 & 9 & 14 & 19 & $(5)$ \\
$\%$ & 9,4 & 17,0 & 26,4 & 35,8 & $(9,4)$ \\
\hline
\end{tabular}

In der nachfolgenden Gesamtübersicht (Tab. 8) sind alle vorkommenden Polychaetenarten, sowohl in horizontaler als auch in vertikaler Ausdehnung angeordnet, noch einmal aufgelistet.

Sowohl aus Tabelle 6 als auch aus der Gesamtübersicht geht hervor, daß nur eine sehr geringe Anzahl von Polychaetenarten ausschließlich im Eulitoral angetroffen wird. Als reine Eulitoralfauna können nur solche Arten gelten, die ausnahmslos die Zonen des Eulitorals bewohnen. Es ist zu folgern, daß die oberste Grenze der Vertikalverbreitung der betreffenden Arten nicht erst im unteren Eulitoral liegt. Zudem sollte die Individuendichte mit zunehmender Tiefe geringer werden, das heißt, das Häufigkeitsmaximum sollte möglichst im oberen Eulitoral liegen.

Unter Zugrundelegung dieser Kriterien sind nach den vorliegenden Befunden lediglich die drei Arten Scolelepis squamata, Microphthalmus sczelkowii und. Ophryotrocha gracilis als Elemente der reinen Eulitoralfauna zu bezeichnen. Alle übrigen, zwar auch im Eulitoral gefundenen Arten reichen - entweder nach eigenen Befunden oder laut Literaturangaben - in ihrer vertikalen Ausdehnung bis ins Sublitoral oder noch tiefer gelegene Zonen hinab. Dies führt zusammen mit dem schon mehrfach erwähnten Faktum, daß die Artenzahl im litoralen Bereich mit zunehmender Tiefe ansteigt, dazu, daß die von Hartmann (1975) für Ostracoden getroffene Feststellung, daß die Fauna des Eulitorals nur eine verarmte Sublitoralfauna darstellt, für die Polychaeten des Helgoländer Felswatts bestätigt werden kann.

Seltene Arten (weniger als 5 Exemplare im gesamten Untersuchungszeitraum) wurden zumeist im Sublitoral bzw. unteren Eulitoral angetroffen. Es handelt sich hierbei um Eteone longa, Eumidia punctifera, Syllides articulocirratus, Sphaerodorum flavum, Malaco- 
ceros vulgaris, Caulleriella caputesocis, Dodecaceria concharum, Zeppelina monostyla, Zeppelina mediopigmentata, Flabelligera affinis, Pherusa flabellata, Pherusa plumosa und Amphitrite figulus.

Ausnahmen bilden lediglich Exogone naidina, Nereis succinea und Polydora ligni, die im mittleren Eulitoral gefunden wurden. Die hier aufgeführten, als "selten" zu bezeichnenden 16 Arten machen 30,2\% der insgesamt gefundenen Arten aus.

Ausgesprochen häufige Arten ( $>50$ gesammelte Exemplare), zu denen Anaitides maculata, Eulalia viridis, Microphthalmus sczelkowii, Typosyllis armillaris, Autolytus prolifer, Nereis pelagica, Nereis virens, Opbryotrocha gracilis, Polydora ciliata, Pygospio elegans, Scolelepis squamata, Malacoceros fuliginosus, Capitella capitata, Capitomastus giardi, Lanice conchilega, Fabricia sabella, Pomatoceros triqueter, Janua pagenstecheri, Spirorbis tridentatus und Spirorbis spirorbis gehören, bilden mit insgesamt 37,7\% den größten Anteil an der Gesamtzahl der gefundenen Arten.

Die restlichen 32,1\% werden von mehr oder weniger regelmäßig im Felswatt anzutreffenden Arten gestellt, die stets nur in geringer Anzahl (insgesamt zwischen $\mathbf{5}$ und 50 Exemplaren) auftraten. Zu ihnen zählen Lepidonotus squamatus, Harmothoë impar, Gattyana cirrosa, Pholoë minuta, Sthenelais boa, Eumidia sanguinea, Kefersteinia cirrata, Syllis gracilis, Typosyllis byalina, Brania cf. pusilla, Sphaerosyllis hystrix, Autolytus edwarsi, Caulleriella bioculata parva, Tharyx multibranchiis, Arenicola marina, Nicolea zostericola und Sabellaria spinulosa.

\section{DISKUSSION DER EINFLUSSE ABIOTISCHER FAKTOREN AUF DIE VERTEILUNG DER POLYCHAETENFAUNA}

Der Nachweis eines direkten Zusammenhanges zwischen einzelnen Faktoren und der Verteilung der Polychaetenfauna läßt sich schwer führen und kann nur für wenige Arten bedingt erbracht werden. Die folgende Diskussion wird sich daher mehr auf allgemeine Aspekte möglicher Einflußnahmen auf die Besiedlung des eulitoralen Helgoländer Felswatts durch Polychaeten beziehen.

\section{Horizontalverteilung}

Uber die Ursachen für die zu verzeichnende allgemeine Zunahme der Polychaetenarten vom östlichen zum westlichen Teil des Untersuchungsgebietes können einige Beobachtungen mitgeteilt werden. Die Änderung im Bestand der Polychaetenfauna geht zum einen mit Veränderungen von Substrateigenschaften einher; die Artenzunahme ist zum anderen mit einer zunehmenden Strukturierung der untersuchten Sammelabschnitte gekoppelt.

Die Änderungender Substrateigenschaften beziehen sich vornehmlich auf die Sauerstoff- bzw. $\mathrm{H}_{2} \mathrm{~S}$-Verhältnisse im Sediment. Sie sind besonders offenkundig im Strandgebiet vor Sammelabschnitt 1 bis zum ersten Sammelabschnitt. Während am östlichen Ende des Strandabschnittes das Sediment gut mit Sauerstoff versorgt ist und $\mathrm{H}_{2} \mathrm{~S}$ vollständig fehlt, kehrt sich dieser Zustand in Richtung auf Sammelabschnitt 1 hin vollkommen um. Im Sammelabschnitt 1 liegen - wie bereits beschrieben - reduzierende Verhältnisse vor. Das 
Tabelle 8

Gesamtübersicht über die im Helgoländer Felslitoral vorkommenden

\begin{tabular}{|c|c|c|c|}
\hline Zone & Vor 1 & Sammelabschnitt 1 & Sammelabschnitt 2 \\
\hline $\begin{array}{l}\text { Oberes } \\
\text { Eulitoral }\end{array}$ & $\begin{array}{l}\text { Anaitides maculata } \\
\text { Nereis virens } \\
\text { Nereis succinea } \\
\text { Malacoceros } \\
\text { fuliginosus } \\
\text { Capitella capitata }\end{array}$ & $\begin{array}{l}\text { Microphtbalmus } \\
\text { sczelkowii } \\
\text { Opbryotrocha gracilis } \\
\text { Scolelepis squamata } \\
\text { Malacoceros fuliginosus } \\
\text { Zeppelina } \\
\text { mediopigmentata } \\
\text { Capitella capitata } \\
\text { Arenicola marina }\end{array}$ & $\begin{array}{l}\text { Microphthalmus } \\
\text { sczelkowoii } \\
\text { Malacoceros } \\
\text { fuliginosus } \\
\text { Capitella capitata } \\
\text { Arenicola marina }\end{array}$ \\
\hline $\begin{array}{l}\text { Mittleres } \\
\text { Eulitoral }\end{array}$ & $\begin{array}{l}\text { Pholoe minuta } \\
\text { Anaitides maculata } \\
\text { Nereis pelagica } \\
\text { Nereis succinea } \\
\text { Nereis virens } \\
\text { Pygospio elegans } \\
\text { Malacoceros } \\
\text { fuliginosus } \\
\text { Capitella capitata } \\
\text { Arenicola marina } \\
\text { Lanice conchilega }\end{array}$ & $\begin{array}{l}\text { Anaitides maculata } \\
\text { Nereis pelagica } \\
\text { Microphthalmus } \\
\text { sczelkowii } \\
\text { Malacoceros } \\
\text { fuliginosus } \\
\text { Capitella capitata } \\
\text { Arenicola marina }\end{array}$ & $\begin{array}{l}\text { Anaitides maculata } \\
\text { Syllis gracilis } \\
\text { Nereis virens } \\
\text { Polydora ligni } \\
\text { Polydora ciliata } \\
\text { Pygospio elegans } \\
\text { Malacoceros } \\
\text { fuliginosus } \\
\text { Capitella capitata } \\
\text { Capitomastus giardi } \\
\text { Lanice conchilega } \\
\text { Fabricia sabella }\end{array}$ \\
\hline & $\begin{array}{l}\text { Nereis virens } \\
\text { Lanice conchilega }\end{array}$ & $\begin{array}{l}\text { Nereis pelagica } \\
\text { Polydora ciliata }\end{array}$ & $\begin{array}{l}\text { Nereis pelagica } \\
\text { Polydora ciliata } \\
\text { Capitomastus giardi } \\
\text { Arenicola marina } \\
\text { Lanice conchilega } \\
\text { Fabricia sabella }\end{array}$ \\
\hline $\begin{array}{l}\text { Unteres } \\
\text { Eulitoral }\end{array}$ & & & \\
\hline
\end{tabular}




\begin{tabular}{|c|c|c|}
\hline Sammelabschnitt 3 & Sammelabschnitt 4 & Sammelabschnitt 5 \\
\hline $\begin{array}{l}\text { Sthenelais boa } \\
\text { Microphthalmus } \\
\text { sczelkowii } \\
\text { Nereis pelagica } \\
\text { Ophryotrocha gracilis } \\
\text { Scolelepis squamata } \\
\text { Malacoceros fuliginosus } \\
\text { Capitella capitata } \\
\text { Arenicola marina }\end{array}$ & $\begin{array}{l}\text { Gattyana cirrosa } \\
\text { Microphthalmus sczelkowii } \\
\text { Sphaerosyllis hystrix } \\
\text { Ophryotrocha gracilis } \\
\text { Polydora ciliata } \\
\text { Pygospio elegans } \\
\text { Capitomastus giardi } \\
\text { Fabricia sabella } \\
\text { Spirorbis tridentatus } \\
\text { Spirorbis spirorbis }\end{array}$ & $\begin{array}{l}\text { Anaitides maculata } \\
\text { Microphthalmus sczelkowii } \\
\text { Sphaerosyllis hystrix } \\
\text { Nereis pelagica } \\
\text { Nereis virens } \\
\text { Ophryotracha gracilis } \\
\text { Polydora ligni } \\
\text { Polydora ciliata } \\
\text { Pygospio elegans } \\
\text { Malacoceros fuliginosus } \\
\text { Capitella capitata } \\
\text { Capitomastus giardi } \\
\text { Arenicola marina }\end{array}$ \\
\hline $\begin{array}{l}\text { Gattyana cirrosa } \\
\text { Sthenelais boa } \\
\text { Anaitides maculata } \\
\text { Eumidia punctifera } \\
\text { Eumidia sanguinea } \\
\text { Microphthalmus sczelkowii } \\
\text { Nereis pelagica } \\
\text { Nereis virens } \\
\text { Polydora ciliata } \\
\text { Scolelepis squamata } \\
\text { Malacoceros fuliginosus } \\
\text { Capitella capitata } \\
\text { Capitomastus giardi } \\
\text { Arenicola marina } \\
\text { Lanice conchilega } \\
\text { Fabricia sabella }\end{array}$ & $\begin{array}{l}\text { Lepidonotus squamatus } \\
\text { Harmothoë impar } \\
\text { Pholoë minuta } \\
\text { Anaitides maculata } \\
\text { Eumidia sanguinea } \\
\text { Eulalia viridis } \\
\text { Syllis gracilis } \\
\text { Typosyllis armillaris } \\
\text { Autolytus prolifer } \\
\text { Nereis pelagica } \\
\text { Opbryotrocha gracilis } \\
\text { Polydora ciliata } \\
\text { Pygospio elegans } \\
\text { Capitomastus giardi } \\
\text { Lanice conchilega } \\
\text { Sabellaria spinulosa } \\
\text { Fabricia sabella } \\
\text { Pomatoceros triqueter } \\
\text { Janua pagenstecheri } \\
\text { Spirorbis tridentatus }\end{array}$ & $\begin{array}{l}\text { Lepidonotus squamatus } \\
\text { Gattyana cirrosa } \\
\text { Pholoë minuta } \\
\text { Anaitides maculata } \\
\text { Syllis gracilis } \\
\text { Typosyllis armillaris } \\
\text { Sphaerosyllis hystrix } \\
\text { Exogone naidina } \\
\text { Autolytus prolifer } \\
\text { Nereis pelagica } \\
\text { Ophryotrocha gracilis } \\
\text { Polydora ciliata } \\
\text { Scolelepis squamata } \\
\text { Malacoceros fuliginosus } \\
\text { Capitomastus giardi } \\
\text { Arenicola marina } \\
\text { Nicolea zostericola } \\
\text { Lanice conchilega } \\
\text { Fabricia sabella } \\
\text { Pomatoceros triqueter } \\
\text { Janua pagenstecberi } \\
\text { Spirorbis tridentatus } \\
\text { Spirorbis spirorbis }\end{array}$ \\
\hline $\begin{array}{l}\text { Sthenelais boa } \\
\text { Anaitides maculata } \\
\text { Nereis pelagica } \\
\text { Nereis virens } \\
\text { Polydora ciliata } \\
\text { Pygospio elegans } \\
\text { Malacoceros fuliginosus } \\
\text { Capitella capitata } \\
\text { Capitomastus giardi } \\
\text { Arenicola marina } \\
\text { Lanice conchilega } \\
\text { Fabricia sabella } \\
\text { Pomatoceros triqueter }\end{array}$ & $\begin{array}{l}\text { Lepidonotus squamatus } \\
\text { Harmothoë impar } \\
\text { Sthenelais boa } \\
\text { Anaitides maculata } \\
\text { Eulalia viridis } \\
\text { Syllis gracilis } \\
\text { Typosyllis armillaris } \\
\text { Brania cf. pusilla } \\
\text { Autolytus prolifer } \\
\text { Autolytus edroarsi } \\
\text { Nereis pelagica } \\
\text { Ophryotrocha gracilis } \\
\text { Polydora ciliata } \\
\text { Malacoceros vulgaris } \\
\text { Tharyx multibranchiis } \\
\text { Arenicola marina } \\
\text { Sabellaria spinulosa }\end{array}$ & $\begin{array}{l}\text { Lepidonotus squamatus } \\
\text { Harmothoë impar } \\
\text { Sthenelais boa } \\
\text { Anaitides maculata } \\
\text { Eulalia viridis } \\
\text { Kefersteinia cirrata } \\
\text { Typosyllis armillaris } \\
\text { Syllides articulocirratus } \\
\text { Brania cf. pusilla } \\
\text { Sphaerosyllis bystrix } \\
\text { Autolytus prolifer } \\
\text { Nereis pelagica } \\
\text { Ophryotrocha gracilis } \\
\text { Polydora ciliata } \\
\text { Pygospio elegans } \\
\text { Tharyx multibranchiis } \\
\text { Dodecaceria concharum }\end{array}$ \\
\hline
\end{tabular}


Tabelle 8 (Fortsetzung)

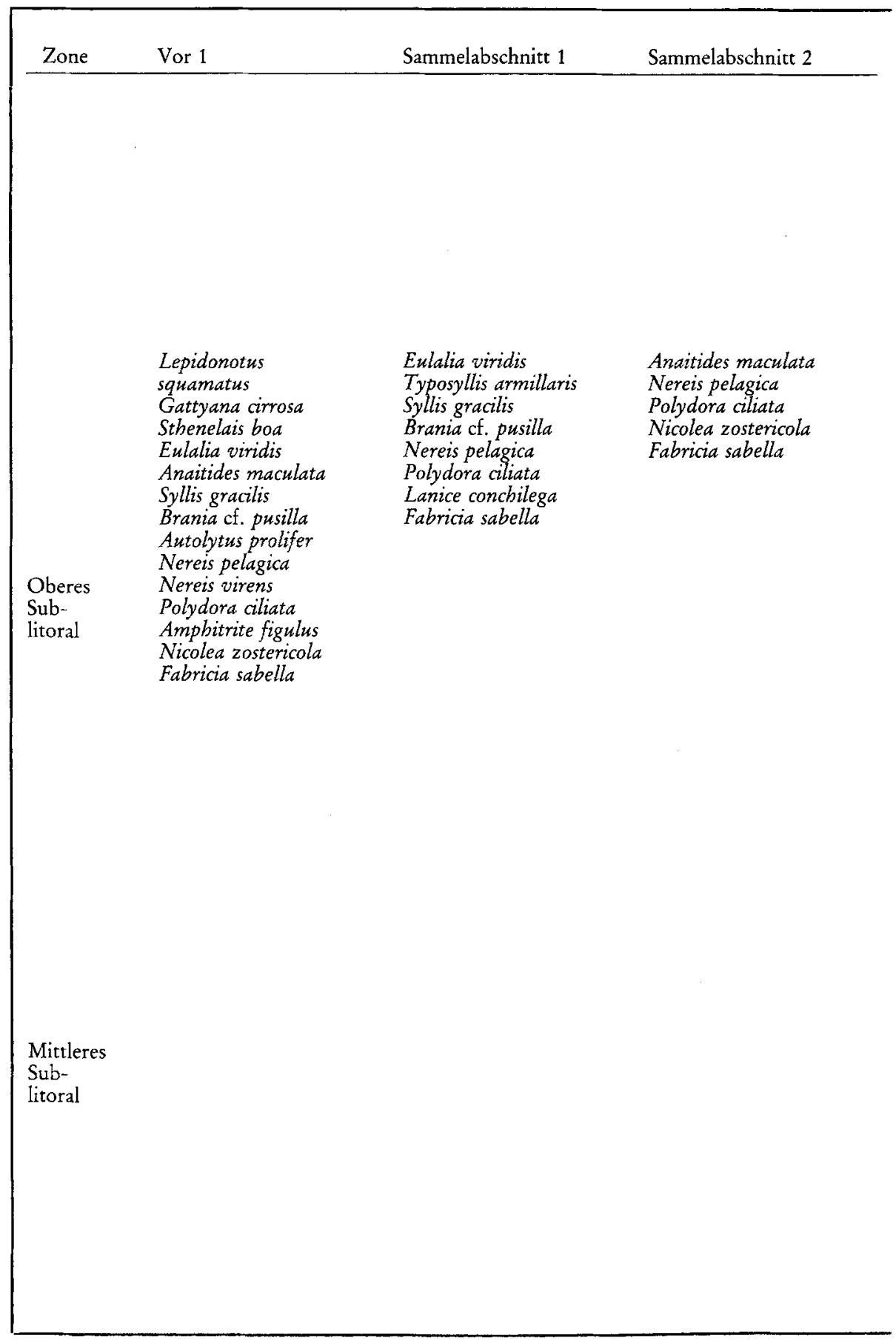


Tabelle 8 (Fortsetzung)

\begin{tabular}{ll} 
Sammelabschnitt 3 & Sammelabschnitt 4 \\
\hline & Fabricia sabella \\
& Pomatoceros triqueter \\
Janua pagenstecheri & Spirorbis tridentatus \\
Spirorbis spirorbis
\end{tabular}

Anaitides maculata

Eulalia viridis

Syllis gracilis

Typosyllis byalina

Typosyllis armillaris

Sphaerosyllis bystrix

Nereis pelagica

Polydora ciliata

Pygospio elegans

Caulleriella bioculata parva

Caulleriella caputesocis

Zeppelina mediopigmentata

Capitomastus giardi

Fabricia sabella
Lepidonotus squamatus

Harmothoë impar

Stbenelais boa

Anaitides maculata

Eumidia punctifera

Eulalia viridis

Syllis gracilis

Typosyllis armillaris

Kefersteinia cirrata

Brania of. pusilla

Sphaerosyllis bystrix

Nereis pelagica

Autolytus prolifer

Autolytus edwarsi

Polydora ciliata

Zeppelina monostyla

Capitomastus giardi

Pherusa flabellata

Nicolea zostericola

Lanice conchilega

Sabellaria spinulosa

Fabricia sabella

Pomatoceros triqueter

Spirorbis tridentatus

Gattyana cirrosa

Syllis gracilis

Typosyllis byalina

Brania cf. pusilla

Sphaerosyllis bystrix

Eulalia viridis

Nereis pelagica

Polydora ciliata

Caulleriella bioculata parva

Capitomastus giardi

Arenicola marina

Nicolea zostericoha

Fabricia sabella

Pomatoceros triqueter
Sammelabschnitt 5

Capitomastus giardi Nicolea zostericola

Lanice conchilega

Sabellaria spinulosa

Fabricia sabella

Pomatoceros triqueter

Spirorbis tridentatus

Spirorbis spirorbis

Lepidonotus squamatus

Harmothoë impar

Gattyana cirrosa

Sthenelais boa

Eteone longa

Anaitides maculata

Eulalia viridis

Syllis gracilis

Typosyllis armillaris

Brania cf. pusilla

Sphaerosyllis bystrix

Autolytus prolifer

Autolytus edwarsi

Nereis pelagica

Polydora ciliata

Caulleriella caputesocis

Tharyx multibranchiis

Pberusa plumosa

Capitomastus giardi

Amphitrite figulus

Nicolea zostericola

Sabellaria spinulosa

Fabricia sabella

Pomatoceros triqueter

Spirorbis tridentatus

Harmotboë impar

Pboloë minuta

Anaitides maculata

Kefersteinia cirrata

Syllis gracilis

Autolytus prolifer

Nereis pelagica

Sphaerodorum flavum

Polydora ciliata

Fabricia sabella 
Sediment ist wegen des hohen $\mathrm{H}_{2} \mathrm{~S}$-Gehalts schwarz gefärbt. Diese Änderung der Sauerstoffverhältnisse im Substrat geht mit einer kontinuierlichen Faunenveränderung einher.

Die im äußersten Osten festzustellenden hohen Individuenzahlen von Nereis virens und Nereis pelagica verringern sich deutlich mit steigendem $\mathrm{H}_{2} \mathrm{~S}-\mathrm{Gehalt}$. Alternierend steigen die im östlichen Teil nur unbedeutend vertretenen Arten Malacoceros fuliginosus und Capitella capitata mit zunehmendem $\mathrm{H}_{2} \mathrm{~S}$-Gehalt stark an. Ein Grund für die ungewöhnlich hohen Individuenzahlen der beiden letztgenannten Arten liegt sicherlich darin, daß es nur sehr wenigen Arten gelungen ist, sich an die extremen Lebensbedingungen im fast azoischen Milieu anzupassen. Die dadurch entfallende mögliche interspezifische Konkurrenz gestattet es in diesem Fall einer bzw. zwei Arten, den gesamten verfügbaren Lebensraum bis an die Grenze der möglichen Besiedlungsdichte zu nutzen.

Andere Faktoren, wie der pH-Wert, die Korngrößen des Substrats, der Detritusgehalt, die Temperatur sowie die Salinität lassen keinen sichtbaren Einfluß auf die Verteilung der aufgeführten Polychaetenarten erkennen.

Ein weiteres Beispiel für die substrateigenschaftsabhängige Verteilung von Polychaetenarten ergibt sich aus dem Vergleich der Sammelabschnitte 1 und 3. Abschnitt 1 ist oben bereits hinreichend charakterisiert worden. Die für das Substrat im Abschnitt 3 typischen Daten sind den Abschnitten "pH-Wert" und "Die Sauerstoffverhältnisse im Sediment" zu entnehmen. Es zeigt sich, daß die wesentlichen Unterschiede zwischen den Sammelabschnitten 1 und 3 zum einen im $\mathrm{pH}$-Wert, zum anderen wiederum im Sauerstoffgehalt des Sediments liegen. Während im Abschnitt 1 die bereits genannten Arten Malacoceros fuliginosus und Capitella capitata dominieren, ist es im Abschnitt 3 Scolelepis squamata.

Nach den bereits erwähnten Untersuchungen von Delamare-Deboutteville (1960), Renaud-Debyser (1963), Giere (1971), Hartwig (1973) und Pfannkuche (1977) entfällt zumindest im hochmarinen Bereich - der $\mathrm{pH}$-Wert als limitierender Faktor für die Verteilung von Organismen. Somit kommt als vermutliche Ursache für die Besiedlung zweier bis auf die Sauerstoffverhältnisse etwa gleichartiger Substrate mit verschiedenen Polychaetenarten wieder der unterschiedliche $\mathrm{O}_{2}$-Gehalt in Betracht.

Scolelepis squamata läßt eine deutliche Präferenz für sauerstoffhaltiges, $\mathrm{H}_{2} \mathrm{~S}$-freies Substrat erkennen, während Malacoceros fuliginosus und Capitella capitata die tiefer gelegenen, $\mathrm{H}_{2} \mathrm{~S}$-haltigen Zonen im Sammelabschnitt 3 bevorzugen. Dies wird auch als Grund dafür angesehen, daß die sonst hauptsächlich im oberen Eulitoral anzutreffenden Arten Malacoceros fuliginosus und Capitella capitatä im Abschnitt 3 ihr Häufigkeitsmaximum ins mittlere bzw. untere Eulitoral verlagern: Im oberen Eulitoral fehlt ihnen der geeignete Lebensraum; für andere Arten nicht besiedelbare, $\mathrm{H}_{2} \mathrm{~S}$-haltige Zonen im Abschnitt 3 sind nur in den tiefer gelegenen Bereichen zu finden.

Für die hier angeführten wenigen Fälle ist es möglich, den offenkundigen Zusammenhang zwischen dem Sauerstoff- bzw. $\mathrm{H}_{2} \mathrm{~S}$-Gehalt des Substrates und der Verteilung bestimmter Polychaetenarten aufzuzeigen.

Im folgenden soll die eingangs erwähnte, von Osten nach Westen zunehmende Strukturierung des Untersuchungsgebietes, die als Hauptursache für die Artenzunahme vermutet wird, noch einmal näher erörtert werden.

Der im Osten an das engere Untersuchungsgebiet angrenzende, relativ einförmige und strukturarme Sandstrand setzt sich im oberen Teil der Sammelabschnitte 1 und 2 fort. Strukturerweiternde Elemente finden sich in diesen Abschnitten nur in tieferen Bereichen 
in Form von Geröll und größeren Gesteinsbrocken, die eine Besiedlung durch andere Arten, als die im strukturarmen Sandstrand vorkommenden, gestatten. Im Sammelabschnitt 3 kommt als weiteres strukturbildendes Element die Miesmuschelbank mit ihren substratgefüllten Spalten und Gezeitentümpeln hinzu. Die zunehmende Strukturierung ist mit einer erhöhten Artenzahl von Polychaeten korreliert.

Die Sammelabschnitte 4 und 5 schließlich sind durch die stark gegliederten und zerklüfteten Klippen charakterisiert, die durch die Schaffung einer großen Anzahl von Kleinbiotopen die günstigsten Voraussetzungen für die Besiedlung durch viele Arten mit unterschiedlichsten ökologischen Ansprüchen bieten. Die große Anzahl von Polychaetenarten in diesem Bereich (vgl. Tab. 8) bestätigt die Richtigkeit dieser Aussage.

Zusammenfassend läßt sich festhalten, daß einförmige, strukturarme Flächen die Ansiedlung weniger Arten mit hohen Individuenzahlen begünstigen, während reich gegliederte Biotope eher für eine Vielzahl von Arten, die gelegentlich allerdings nur durch wenige Vertreter repräsentiert sind, geeignet erscheinen.

\section{Vertikalverteilung}

Die allgemein zu beobachtende Zunahme der Artenzahlen vom oberen Eulitoral zum mittleren Sublitoral ist in auffälliger Weise mit einem zeitlichen Anstieg der Dauer der täglichen Wasserbedeckung verbunden. Es liegt daher nahe, die Wassertiefe als einen der Hauptfaktoren für die deutliche vertikale Zonierung der Anzahl der Polychaetenarten anzunehmen.

Der Einfluß weiterer Faktoren, wie Temperatur und Salzgehalt auf die vertikale Verteilung von Organismen ist bekannt (Hartmann-Schröder \& Hartmann, 1962; Hartmann, 1975), konnte jedoch in dem räumlich eng begrenzten Untersuchungsgebiet nicht nachgewiesen werden. Fest steht, daß wärmeliebende Arten auf die oberen Bereiche des Litorals beschränkt bleiben, während - großräumig betrachtet - kälteliebende Elemente in tieferen kalten Wasserkörpern weit in wärmere Klimabereiche vordringen können (Hartmann, 1975).

Der Salzgehalt als beeinflussender Faktor der Vertikalverteilung spielt vor allem in den oberen Litoralbereichen eine Rolle, die täglich mehrere Stunden lang vom Wasser unbedeckt sind. Hier kann es durch Niederschläge einerseits zu einem Absinken des Salzgehaltes kommen, andererseits kann eine hohe Verdunstungsrate z. B. in stehenbleibenden kleinen Gezeitentümpeln eine plötzliche beträchtliche Erhöhung des Salzgehaltes bewirken. Das Litoral, einschließlich des oberen Sublitorals, wird daher hauptsächlich von einer euryhalinen Fauna bewohnt, während stenohaline Arten tiefere Zonen bevorzugen.

Abschließend sei kritisch angemerkt, daß aus dem komplizierten Gesamtgefüge sich gegenseitig in bislang noch weitgehend ungeklärter Weise beeinflussender ökologischer Faktoren nur einige wenige, leicht erfaßbare Größen herausgegriffen und zur Interpretation der Verteilung von Polychaeten im Helgoländer Felslitoral herangezogen werden konnten.

Um zu einer aussagekräftigen Analyse des ökologischen Faktorengefüges zu kommen, das die Lebensweise einer oder mehrerer Arten bestimmt, müßten aufwendige experimentelle Freiland- und Laboruntersuchungen durchgeführt werden, wie sie z. B. von Klöckner (1976) für Pomatoceros triqueter begonnen wurden. 


\section{ZOOGEOGRAPHISCHE ANMERKUNGEN}

Von den 53 im Helgoländer Felslitoral gefundenen Polychaetenarten sind zwei sowie eine Unterart neu (Syllides articulocirratus n. sp., Zeppelina mediopigmentata n. sp. und Caulleriella bioculata parva n. ssp.) (Gillandt, 1979). Außer ihnen ist lediglich Microphthalmus sczelkowii bisher ausschließlich in der Nordsee gefunden worden. Acht weitere Arten sind erstmalig in der Deutschen Bucht nachgewiesen worden. Es handelt sich um Syllis gracilis, Typosyllis byalina, Brancia cf. pusilla, Sphaerosyllis bystrix, Tharyx multibranchiis, Zeppelina monostyla, Pherusa flabellata und Spirorbis tridentatus.

Ihr Auftreten in Helgoland hängt aller Wahrscheinlichkeit nach mit dem felsigen Untergrund zusammen, der in dieser Form in der Deutschen Bucht nur im Helgoländer Litoral ausgeprägt ist.

Die meisten Polychaetenarten sind sehr weit verbreitet. Es ist daher nur in wenigen Fällen möglich, von reinen Kalt- oder Warmwasserformen zu sprechen und einen etwa vorhandenen arktischen oder lusitanischen Einfluß nachzuweisen.

$\mathrm{Zu}$ ausgesprochen wärmeliebenden Vertretern können die zirkumtropisch-borealantiboreal verbreiteten Arten Sthenelais boa, Eumidia sanguinea, Syllis gracilis, Nereis succinea, Polydora ciliata und Scolelepis squamata gerechnet werden. Kälteliebende Elemente der Helgoländer felslitoralen Polychaetenfauna sind die arktisch-amphiboreal verbreiteten Arten Gattyana cirrosa und Nicolea zostericola.

Alle übrigen bei Helgoland gefundenen Arten umfassen in ihrer Verbreitung derartig unterschiedliche Klimabereiche, daß sie als mehr oder weniger eurytherme Formen als Indikatoren für bestimmte Klimaeinflüsse kaum geeignet sind.

Danksagungen. Frau Dr. habil. G. Hartmann verdanke ich die Anregung zur Bearbeitung des vorliegenden Themas. Ihr und Herrn Prof. Dr. G. Hartmann danke ich für das stete Interesse, mit dem sie den Fortgang der Arbeit in zahlreichen Gesprächen und Diskussionen verfolgten. Bei Herrn Prof. Kinne bedanke ich mich für die Bereitstellung eines Arbeitsplatzes in der Biologischen Anstalt Helgoland. Den Mitarbeitern der "Arbeitsgruppe Gastforschung" der Biologischen Anstalt Helgoland danke ich für ihre Unterstützung und Hilfsbereischaft während der Zeit der Probeentnahmen auf Helgoland.

\section{ZITIERTE LITERATUR}

Amanieu, M., 1969. Recherches écologiques sur les faunes des plages arbiteés de la region d'Arcachon. Helgoländer wiss. Mceresunters. 19, 455-577.

Amoureux, L., 1966. Etude bionomique et écologique de quelques Annélides Polychètes des sables intertidaux des côtes Ouest de la France. Archs Zool. exp. gén. 107, 1-218.

— \& Katzmann, W., 1971. Note faunistique et écologique sur une collection d'Annélidés Polychètes de substrats rocheux circalittoraux de la région de Rovinj (Yougoslavie). Zool. Anz. 186, 115-122.

Attems, C., 1896. Beiträge zur Meeresfauna von Helgoland. VI. Beitrag zur Kenntnis der rhabdocoelen Turbellarien Helgolands. Wiss. Meeresunters. (Abt. Helgoland) 2 (1), 219-232.

Baas-Becking, L. G. M. \& Wood, E. J., 1955. Biological processes in the estuarine environment. I, II. - Ecology of the sulphur cycle. Proc. K. ned. Akad. Wet. (B) 58, 160-181.

Bellan, G., 1969. Polychétes des horizons supérieurs de l'étage infralittoral rocheux dans la région Provençale. Tethys 1, 349-366. 
Bruce, J. R., Colman, J. S. \& Jones, N. S., 1963. Marine fauna of the Isle of Man. Univ. Press, Liverpool, $307 \mathrm{pp}$.

Caspers, H., 1939. Die Bodenfauna der Helgoländer Tiefen Rinne. Helgoländer wiss. Meeresunters. 2, $1-112$.

- 1950. Die Lebensgemeinschaft der Helgoländer Austernbank. Helgoländer wiss. Meeresunters. 3, 120-169.

Dalla-Torre, K. W. v., 1889. Die Fauna von Helgoland. Zool. Jb. (Suppl.) 2, 1-99.

Delamare-Deboutteville, C., 1960. Biologie des eaux souterraines littorales et continentales. Vie Milieu. (Suppl.) 9, 1-740.

Dietrich, R., Höhnk, W. \& Manzel, W.-D., 1965. Studien zur Chemie ozeanischer Bodenproben, III. Beziehungen zwischen physikalischen und chemischen Größen in den Böden der Nordsee und der Außenweser. Veröff. Inst. Meeresforsch. Bremerh. 9, 242-278.

Dörjes, J., 1968. Zur Okologie der Acoela (Turbellaria) in der Deutschen Bucht. Helgoländer wiss. Meeresunters. 18, 78-115.

Ehrenbaum, E., 1896. Beiträge zur Meeresfauna von Helgoland. VIII. Die Cumaceen und Schizopoden von Helgoland nebst neueren Beobachtungen über ihr Vorkommen in der Deutschen Bucht und in der Nordsee. Wiss. Meeresunters. (Abt. Helgoland) 2 (1), 403-435.

Eliason, A., 1920. Biologisch-faunistische Untersuchungen aus dem Oresund. Polychaeta. Acta Univ. Lund. (Avd. 2) 16 (6), 1-103.

Enckell, P. H., 1968. Oxygen availibility and microdistribution of interstitial mesofauna in Swedish freshwater sandy beaches. Oikos 19, 271-291.

Fauvel, P., 1923. Polychètes errantes. Faune Fr. 5, 1-488.

- 1927. Polychètes sédentaire. Addenda aux errantes, Archiannélides, Myzostomaires. Faune Fr. 16, $1-494$.

Fenchel, T., 1969. The ecology of marine microbenthos IV. Structure and function of the bentic ecosystem, it's chemical and physical factors and the microfauna communities with special reference to the ciliated protozoa. Ophelia 6, 1-182.

- 1971. The reduction-oxidation properties of marine sediments and the vertical distribution of the microfauna. Vie Milieu. (Suppl.) 22, 509-521.

- \& Jansson, B.-O., 1966. On the vertical distribution of the microfauna in the sediments of a brackishwater beach. Ophelia 3, 161-177.

- Jansson, B.-O. \& von Thun, W., 1967. Vertical and horizontal distribution of the metazoan microfauna and of some physical factors in a sandy beach in the northern part of the Øresund. Ophelia 4, 227-243.

- \& Riedl, R. J., 1970. The sulfide system: a new biotic community underneath the oxidized layer of marine sand bottoms. Mar. Biol. 7, 255-268.

Friedrich, H., 1938. Polychaeta. Tierwelt Nord- u. Ostsee. 32 (6b), 1-210.

George, J. D., 1974. The marine fauna of Lundy Polychaeta (Marine Bristleworms). Rep. Lundy Fld Soc. 25, 33-48.

Gerlach, S. A., 1953. Die biozönotische Gliederung der Nematodenfauna an den deutschen Küsten. Z. Morph. Okol. Tiere 41, 411-512.

Gibbs, P. E., 1969. A quantitative study of the polychaete fauna of certain fine deposits in Plymouth Sound. J. mar. biol. Ass. U. K. 49, 311-326.

Giere, O., 1971. Beziehungen zwischen abiotischem Faktorensystem, Zonierung und Abundanz mariner Oligochaeten in einem Küstengebiet der Nordsee. Thalassia jugosl. 7, 67-77.

- 1973. Oxygen in the marine hygropsammal and the vertical microdistribution of oligochaetes. Mar. Biol. 21, 180-189.

Gillandt, L., 1979. Zur Systematik, Biologie und Autökologie der Polychaeten des Helgoländer Felslitorals. Mitt. hamb. zool. Mus. Inst. 76 (im Druck).

Gruet, Y., 1974. Macrofaune des substrats meubles intertideaux entre le pont d'yeu et Sion-surL'océan (Vendee). Bull. Soc. Sci. nat. Oest Fr. 72 (3), 12-28.

Hagmeier, A., 1930. Die Besiedlung des Felsstrandes und der Klippen von Helgoland. Teil I. Der Lebensraum. Wiss. Meeresunters. (Abt. Helgoland) 15 (4), 1-35.

Hamond, R., 1966. The Polychaeta of the coast of Norfolk. Cah. Biol. mar. 7, 383-436. 
Hartlaub, C., 1896a. Beiträge zur Meeresfauna von Helgoland. IV. Die Coelenteraten Helgolands. Wiss. Meeresunters. (Abt. Helgoland) 1 (1), 161-206.

- 1896b. Beiträge zur Meeresfauna von Helgoland. X. Die Hydromedusen Helgolands. Wiss. Meeresunters. (Abt. Helgoland) 2 (1), 449-536.

Hartmann, G., 1975. Ostracoda. Bronns Kl. Ordn. Tierreichs, 5 (1. Abt., 2. Buch, T. 4, Lfg. 4), 569-786.

Hartmann-Schröder, G., 1960. Polychaeten aus dem Roten Meer. Kieler Meeresforsch. 16, 69-125.

- 1971. Annelida, Borstenwlirmer, Polychaeta. Tierwelt Dtl. 58, 1-594.

- \& Hartmann, G., 1962. Zur Kenntnis des Eulitorals der chilenischen Pazifikküste und der argentinischen Küste Südpatagoniens unter besonderer Berücksichtigung der Polychaeten und Ostracoden. Mitt. hamb. zool. Mus. Inst. 60 (Ergbd.), 5-270.

- \& Stripp, K., 1968. Beiträge zur Polychaetenfauna der Deutschen Bucht. Veröff. Inst. Meeresforsch. Bremerh. 11, 1-24.

Hartmeyer, R., 1908. Beiträge zur Meeresfauna von Helgoland. XIV. Die Ascidien von Helgoland. Wiss. Meeresunters. (Abt. Helgoland) 8 (1), 117-127.

Hartwig, E., 1973. Die Ciliaten des Gezeitenstrandes der Nordseeinsel Sylt. II. Okologie. Mikrofauna des Meeresbodens 21, 1-171.

Heincke, F., 1896a. Beiträge zur Meeresfauna von Helgoland. I. Die Fische Helgolands. Wiss. Meeresunters. (Abt. Helgoland) 1 (1), 99-120.

- 1896b. Beiträge zur Meerèsfauna von Helgoland. II. Die Mollusken Helgolands. Wiss. Meeresunters. (Abt. Helgoland) 1 (1), 121-153.

- 1896c. Beiträge zur Meeresfauna von Helgoland. VII. Nachträge zur Fisch- und Molluskenfauna Helgolands I. Wiss. Meeresunters. (Abt. Helgoland) 2 (1), 233-252.

Jansson, B.-O., 1967. The availability of oxygen for the interstitial fauna of sandy beaches. J. exp. mar. Biol. Ecol, 1, 123-143.

Kirkegaard, J. B., 1969. A quantitative investigation of the central North Sea Polychaeta. Spolia zool. Mus. haun. 29, 1-285.

Klöckner, K., 1976. Zur Okologie von Pomatoceros triqueter (Serpulidae, Polychaeta). Helgoländer wiss. Meeresunters. 28, 352-400.

Krumbein, W. E., 1975. Verwitterung, Abtragung und Küstenschutz auf der Insel Helgoland. Abh. Verh. naturw. Ver. Hamburg (NF) 18-19, 5-31.

- 1977. Zur Frage der Verwitterung der Felsmasse der Insel Helgoland. Abh. Verh. naturw. Ver. Hamburg 20, 5-12.

Lauterborn, R., 1896. Beiträge zur Meeresfauna von Helgoland. V. Die pelagischen Protozoen und Rotatorien Helgolands. Wiss. Meeresunters. (Abt. Helgoland) 1 (1), 207-213.

Lemon, E. R. \& Erickson, A. E., 1952. The measurement of oxygen diffusion in the soil with a platinum microelectrode. Soil Sci. Soc. Am. Proc. 16, 160-163.

- 1954. Priciple of the platinum microelectrode as a method of characterizing soil aeration. Soil. Sci. 79, 383-392.

Linke, O., 1939. Die Biota des Jadebusenwattes. Helgoländer wiss. Meeresunters. 1, 201-348.

Michaelis, H., 1970. Biologische Untersuchung der Watten und Landgewinnungsfelder bei Schillighorn. Jber. ForschSt. Norderney 20, 61-76.

- 1975. Die Makrofauna des nördlichen Eversandes (Wesermündung). Jber. ForschSt. Norderney 27, 167-179.

Michaelsen, W., 1897. Die Polychaetenfauna der deutschen Meere einschließlich der benachbarten und verbindenden Gebiete. Wiss. Meeresunters. (Abt. Helgoland) 2 (1), 7-217.

Mortensen, T., 1922. Biologiske Studier over Sandstrandsfaunaen, saerlig ved de danske Kyster. Vidensk. Meddr dansk naturh. Foren. 74, 23-56.

Nienburg, W., 1930. Die Besiedlung des Felsstrandes und der Klippen von Helgoland. Teil II. Die Algen. Wiss. Meeresunters. (Abt. Helgoland) 15, 1-15.

Orrhage, L., 1967. Über die Anatomie des zentralen Nervensystems der sedentären Polychaeten. Ark. Zool. 19 (3), 99-133.

Pfannkuche, O., 1977. Okologische und systematische Untersuchungen an naidomorphen Oligochaeten brackiger und limnischer Biotope. Diss. Univ. Hamburg, 138 pp. 
Remane, A., 1940. Einführung in die zoologische Okologie der Nord- und Ostsee. Tierwelt Nord-u. Ostsee 34 (1a), 1-238.

- 1955. Die Brackwasser-Submergenz und die Umkompensation der Coenosen in Belt und Ostsee. Kieler Meeresforsch. 11, 59-73.

Renaud-Debyser, J., 1963. Recherches écologiques sur la fauna interstitielle des sables (Basin d'Arcachon, Ile de Bimini, Bahamas). Vie Milieu (Suppl.) 15, 1-157.

Schmidt, H. E. \& Machan, R., 1975. Eh-measurements in marine sediments under laboratory conditions. - Cah. Biol. mar. 16, 733-741.

Schmidt, P., 1968. Die quantitative Verteilung und Populationsdynamik des Mesopsammons am Gezeitensandstrand der Nordseein'sel Sylt. I. Faktorengefüge und biologische Gliederung des Lebensraumes. Int. Revue ges. Hydrobiol. 53, 723-779.

Schmidt-Thomé, P., 1937. Der tektonische Bau und die morphologische Gestaltung von Helgoland auf Grund einer Untersuchung der kleintektonischen Erscheinungsformen. Abh. Verh. naturw. Ver. Hamburg 1-2, 215-249.

Schuster, O., 1951. Die Lebensgemeinschaften auf dem Südwatt der Nordsee-Insel Mellum. Senckenbergiana 32, 49-65.

Skaumal, U., 1977. Zur Kenntnis der Ostracoden des Felslitorals von Helgoland. Diss. Univ. Hamburg, 98 pp.

Sokolowsky, A., 1900. Beiträge zur Meeresfauna von Helgoland. XI. Die Amphipoden Helgolands. Wiss. Meeresunters. (Abt. Helgoland) 4 (1), 141-166.

Stephenson, T. A. \& Stephenson, A., 1972. Life between tidemarks on rocky shores. Freeman, San Francisco, 425 pp.

Stripp, K., 1969a. Jahreszeitliche Fluktuationen von Makrofauna und Meiofauna in der Helgoländer Bucht. Veröff. Inst. Meeresforsch. Bremerh. 12, 65-94.

- 1969b. Die Assoziationen des Benthos in der Helgoländer Bucht. Veröff. Inst. Meeresforsch. Bremerh. 12, 95-141.

- 1969c. Das Verhältnis von Makrofauna und Meiofauna in den Sedimenten der Helgoländer Bucht. Veröff. Inst. Meeresforsch. 12, 143-148.

Timm, R., 1896. Beiträge zur Meeresfauna von Helgoland. III. Die Copepoden und Cladoceren Helgolands. Wiss. Meeresunters. (Abt. Helgoland) 1 (1), 155-159.

Trask, P. D., 1932: Origin and environment of source sediments of petroleum. Gulf Publs, Houston, $67 \mathrm{pp}$.

Weltner, W., 1896. Beiträge zur Meeresfauna von Helgoland. IX. Die Cirripedien Helgolands. Wiss. Meeresunters. (Abt. Helgoland) 2 (1), 437-447.

Westheide, W., 1966. Zur Polychaetenfauna des Eulitorals der Nordseeinsel Sylt. Helgoländer wiss. Meeresunters. 13, 203-209.

- 1967. Monographie der Gattungen Hesionides Friedrich und Microphthalmus Mecznikow (Polychaeta, Hesionidae). Z. Morph. Tiere 61, 1-159.

- 1972. Räumliche und zeitliche Differenzierungen im Verteilungsmuster der marinen Interstitialfauna. Verh. dt. zool. Ges. 65, 23-32.

Whitfield, M., 1969. Eh as an operational parameter in estuarine studies. Limnol. Oceanogr. 14, 547-558.

Wieser, W., 1975. The meiofauna as a tool in the study of habitat hererogenity: Ecophysiological aspects. A review. Cah. Biol. mar. 26, 647-670.

Wohlenberg, E., 1937. Die Wattenmeer-Lebensgemeinschaften im Königshafen von Sylt. Helgoländer wiss. Meeresunters. 1 (1), 1-92.

Wood, E. J., 1965. Marine microbial ecology. Chapmann \& Hall, London, 244 pp.

ZoBell, C. E., 1946. Studies on redoxpotential of marina sediment. Bull. Am. Ass. Petrol. Geol. 30, $477-513$. 